\title{
Complexing Behaviour and Antifungal Activity of N-[(1E)-1-(1H-Benzimidazol-2-yl)ethylidene]morpholine-4- carbothiohydrazide and Related Ligand with Metal Ions
}

\author{
Madhu Bala ${ }^{1,2}$ and L. K. Mishra ${ }^{3}$ \\ ${ }^{1}$ Department of Chemistry, N.I.T, Patna, Bihar 800005, India \\ ${ }^{2}$ D/O Bansropan Sharma, Moti Chowk Saidpura, Thana Road Khagaul, Patna, Bihar 801105, India \\ ${ }^{3}$ Department of Chemistry, Patna University, Patna, Bihar 800005, India \\ Correspondence should be addressed to Madhu Bala; madhub252@gmail.com
}

Received 6 September 2013; Revised 11 December 2013; Accepted 17 December 2013; Published 22 April 2014

Academic Editor: Alfonso Castiñeiras

Copyright ( $) 2014$ M. Bala and L. K. Mishra. This is an open access article distributed under the Creative Commons Attribution License, which permits unrestricted use, distribution, and reproduction in any medium, provided the original work is properly cited.

\begin{abstract}
The coordination complexes of bivalent metal ions with N-[(1E)-1-(1H-Benzimidazol-2-yl)ethylidene $]$ morpholine4-carbothiohydrazide ( $\mathrm{H}_{2}$ bmctz, $\left.\quad \mathrm{H}_{2} \mathrm{~L}-1\right)$ and $\mathrm{N}$-[(1E)-1-(1H-Benzimidazol-2-yl)(phenyl)methylidene] morpholine-4carbothiohydrazide $\left(\mathrm{H}_{2}\right.$ bpmctz, $\left.\mathrm{H}_{2} \mathrm{~L}-2\right)$ were prepared and their neutral, monoanionic, and dianionic forms of ligands of compositions $\left[\mathrm{M}\left(\mathrm{H}_{2} \mathrm{~L}\right) \mathrm{X}_{2}\right]\left(\mathrm{M}=\mathrm{Co}^{\mathrm{II}}, \mathrm{Ni}^{\mathrm{II}}, \mathrm{Cu}^{\mathrm{II}}, \mathrm{Zn}^{\mathrm{II}}, \mathrm{Cd}^{\mathrm{II}}\right.$, or $\mathrm{Pd}^{\mathrm{II}}, \mathrm{X}=\mathrm{Cl}^{-}$or $\mathrm{Br}^{-}$, and $\mathrm{H}_{2} \mathrm{~L}=\mathrm{H}_{2} \mathrm{~L}-1$ or $\left.\mathrm{H}_{2} \mathrm{~L}-2\right),\left[\mathrm{M}(\mathrm{HL})_{2}\right] n \mathrm{H}_{2} \mathrm{O}$ where $\left(\mathrm{M}=\mathrm{Co}^{\mathrm{II}}, \mathrm{Cu}^{\mathrm{II}}, \mathrm{Mn}^{\mathrm{II}}, \mathrm{Ni}^{\mathrm{II}}, \mathrm{Zn}^{\mathrm{II}}\right.$, or $\mathrm{Cd}^{\mathrm{II}}, \mathrm{H}_{2} \mathrm{~L}=\mathrm{H}_{2} \mathrm{~L}-1$ or $\mathrm{H}_{2} \mathrm{~L}-2$, and $n=0$ or 2$)$, and $[\mathrm{MLB}] n \mathrm{~B}\left(\mathrm{M}^{\mathrm{C}} \mathrm{Cu}^{\mathrm{II}}, \mathrm{Ni}^{\mathrm{II}}, \mathrm{Zn}^{\mathrm{II}}, \mathrm{Cd}^{\mathrm{II}}, \mathrm{or}\right.$ $\mathrm{Pd}^{\mathrm{II}}, \mathrm{B}=\mathrm{H}_{2} \mathrm{O}$, Py, or $Y$-pic, $n=0$ and $n=1$ if $\mathrm{B}=\mathrm{H}_{2} \mathrm{O}$ for $\mathrm{Ni}(\mathrm{II})$ and $\mathrm{H}_{2} \mathrm{~L}=\mathrm{H}_{2} \mathrm{~L}-1$ or $\mathrm{H}_{2} \mathrm{~L}-2$ ) have been characterised by magnetic susceptibility measurements, electrical conductance values and spectral properties. The magnetic moment value of $\left[\mathrm{M}\left(\mathrm{HL} \mathrm{L}_{2}\right)\right]$ $\left(\mathrm{M}=\mathrm{Mn}^{\mathrm{II}}, \mathrm{Ni}^{\mathrm{II}}, \mathrm{Fe}^{\mathrm{II}}\right.$ or $\left.\mathrm{Cu}^{\mathrm{II}}\right)$ type complexes is consistent with high spin octahedral structure while those of $\left[\mathrm{M}\left(\mathrm{H}_{2} \mathrm{~L}\right) \mathrm{X}_{2}\right]\left(\mathrm{M}=\mathrm{Co}{ }^{\mathrm{II}}\right.$, $\mathrm{Ni}^{\mathrm{II}}, \mathrm{Cu}^{\mathrm{II}}, \mathrm{Zn}^{\mathrm{II}}$, or $\mathrm{Cd}^{\mathrm{II}}, \mathrm{X}=\mathrm{Cl}^{-}$or $\mathrm{Br}^{-}$) possess five coordinated trigonal bipyramidal geometry. The adduct complexes [MLB] $n \mathrm{~B}$ $\left(\mathrm{M}=\mathrm{Ni}^{\mathrm{II}}\right.$ or $\mathrm{Cu}^{\mathrm{II}}, \mathrm{B}=\mathrm{H}_{2} \mathrm{O}, \mathrm{Py}$, or $Y$-pic) are four coordinated planar and those of $\mathrm{Zn}^{\mathrm{II}}$ and $\mathrm{Cd}$ (II complexes $[\mathrm{MLB}],\left(\mathrm{H}_{2} \mathrm{~L}=\mathrm{H}_{2} \mathrm{~L}-1\right.$ or $\mathrm{H}_{2} \mathrm{~L}-2, \mathrm{~B}=\mathrm{H}_{2} \mathrm{O}$, Py or $Y$-pic) are tetrahedral. These ligands have been suggested to coordinate as tridentate (N N S) donor molecule in complexes of type $\left[\mathrm{M}\left(\mathrm{H}_{2} \mathrm{~L}\right) \mathrm{X}_{2}\right],\left[\mathrm{M}(\mathrm{HL})_{2}\right]$, and $[\mathrm{MLB}]$. The antifungal activity of ligands and some of their metal complexes were studied and it was observed that metal complexes show higher activity than free ligand.
\end{abstract}

\section{Introduction}

Preparation and structural aspects of various metal ions with a number of benzimidazole derivatives have been reported by one of us and a number of chemists [1-10]. Medicinal properties of benzimidazole derivatives have established that benzimidazoles have privileged substructures for drug design $[11,12]$. The most vital benzimidazole derivatives is $\mathrm{N}$ ribosyl-5,6-dimethylbenzimidazole which is axial ligand in vitamin $B_{12}$ possessing selective neuropeptide YY1 receptor antagonists [13], 5-lipo xyginase inhibitor [14] and poly (ADP-ribose) polymerase inhibitors [14]. Benzimidazole derivatives are of immense interest because of their wide spectrum of biological activity such as anticancer [15], antiviral [16, 17], antihistaminic [18], antifungal [19], antiHBV [20], antibacterial [21], antitumoral [22], antiparasitic [22], antihelminitic [23], anti-inflammatory, local analgesic, hypotensive [24], antiulcer [25], and neuro leptic cardiotonic [26]. Extensive biochemical and pharmacological studies have confirmed that various benzimidazole derivatives are effective against various strains of microorganisms [25]. The wide ranging biological and industrial interest intrigued chemists to explore new benzimidazole derivatives and their metal complexes which might have novel mechanisms of action and drug activity [15-25]. The metal ions selected for present studies $\mathrm{Mn}(\mathrm{II}), \mathrm{Co}(\mathrm{II}), \mathrm{Cu}(\mathrm{II}), \mathrm{Fe}(\mathrm{II}), \mathrm{Ni}(\mathrm{II}), \mathrm{Zn}(\mathrm{II})$, 
and Cd(II) are versatile essential micronutritents in biological systems [27-32]. Copper is crucial for biological function of several enzymes and proteins, in energy metabolism, antioxidant, and mitochondrial respiration [27]. Zinc(II) is essential for nucleic acid synthesis, cell growth division, and differentiation and its deficiency causes abnormalities in function of cell carboxypeptidase, amino peptidase, carbonic anhydrase, DNA and RNA polymerase. Zinc is also essential for life and it regulates the function of genes in the nuclei of thecell (Lipsocomb and Starter, 1996). Iron is the most vital element for life and iron-sulphur proteins formation (Richardson, 2002). Manganese is a component of nucleic acid and it accelerates the synthesis of cholesterol. Manganese enzymes are involved in metabolic pathway of DNA synthesis, sugar metabolism and protein modification. Cobalt is a component of vitamin $\mathrm{B}_{12}$ and enzyme nutritional cofactor necessary for the formation of red blood cell [31]. Nickel is remarkably useful metal in biological chemistry. It modifies antioxidant system and is associated with chromosomal aberrations and mutation [31]. In continuation of our work on coordinating behaviour of benzimidazole and imidazole derivatives with various metal ions [2-6], and biological significance of above metal ions, we report in present communication, the preparation, characterization, and antifungal activity of complexes of these metals with of $\mathrm{N}$-[(1E)-1-(1H-benzimidazol-2-yl)ethylidene]morpholine4-carbothiohydrazide $\left(\mathrm{H}_{2} \mathrm{~L}-1\right)$ and $\mathrm{N}$-[(1E)-1- $(1 \mathrm{H}-\mathrm{ben}-$ zimidazol-2-yl)(phenyl)methylidene] morpholine-4-carbothiohydrazide $\left(\mathrm{H}_{2} \mathrm{~L}-2\right)$.

\section{Material and Methods}

The metal salts used were usually BDH Anal-R chemicals or E. Merk Extra pure reagent. Palladium(II) chloride was obtained from Johnson Matthey London. Solvents and organic reagent, hydrazine, morpholine, carbon disulphide, Chloroacetic acid were obtained from E. Merk, Nice, Sd. Fine and Ranbaxy. The metal contents of complexes were estimated by standard method sulphur content was determined as sulphate, and nitrogen content was estimated by Nitrometer (Duma's method) in the laboratory. The infrared spectra of complexes were recorded on Shimadzu-FTIR spectrometer at IIT patna. The results of $\mathrm{C}, \mathrm{H}$, and $\mathrm{N}$ were obtained from CDRI Lucknow. The results of mass spectrum and U-V absorption spectrum of ligand and some complexes were obtained from IIT patna. The ${ }^{1} \mathrm{HNMR}$ spectrums were recorded at CDRI Lucknow. The magnetic susceptibilities of complexes were determined by Gouy method at room temperature. The electrical conductance values of complexes were determined in DMF on Systonic conductivity Meter Bridge at $30-31^{\circ} \mathrm{C}$. The results of measurements are given in Tables 1 and 2 .

Ligand molecules $\left(\mathrm{H}_{2}\right.$ bmctz, $\mathrm{H}_{2} \mathrm{~L}-1$ or $\mathrm{H}_{2}$ bpmctz, $\left.\mathrm{H}_{2} \mathrm{~L}-2\right)$ were prepared by condensing 2-acetylbenzimidazole or 2-benzoylbenzimidazole [33] with morpholine-Nthiohydrazide [34] in aqueous ethanol containing a little amount of acetic acid. 2-Acetylbenzimidazole or 2benzoylbenzimidazole in situ was prepared by oxidation of
1-(1H-benzimidazol-2-yl)ethanol and 1-(1H-benzimidazol2-yl)phenylmethanol [35] with chromic acid [33]. The 0.1 mole of 2-acetylbenzimidazole in $100 \mathrm{~mL}$ aqueous ethanol containing $2 \mathrm{~mL}$ acetic acetic acid was refluxed with 0.12 mole of aqueous ethanolic solution of morpholine$\mathrm{N}$-thiohydrazide(morpholine-4-carbothiohydrazide) on a steam bath for two hours. The ligand separated as cream of yellow product. The ligands were analysed. For $\mathrm{H}_{2}$ bmctz $\left(\mathrm{H}_{2} \mathrm{~L}-1\right) \quad\left(\mathrm{C}_{14} \mathrm{H}_{17} \mathrm{~N}_{5} \mathrm{SO}\right)$ nitrogen was found $23.06 \%$ calculated value is $23.11 \%$. M.pt recorded, $197^{\circ} \mathrm{C}$ (uncorrected). The value of nitrogen found for $\mathrm{H}_{2}$ bpmctz $\left(\mathrm{H}_{2} \mathrm{~L}-2\right)$ is $19.01 \%$, and that calculated for $\mathrm{H}_{2} \mathrm{~L}-2$ $\left(\mathrm{C}_{19} \mathrm{H}_{19} \mathrm{~N}_{5} \mathrm{SO}\right)$ is $19.18 \%$, M.pt recorded $242^{\circ} \mathrm{C}$ (uncorrected).

2.1. Preparation of Complexes $\left[\mathrm{M}\left(\mathrm{H}_{2} L\right) \mathrm{X}_{2}\right]\left(\mathrm{M}=\mathrm{Cu}{ }^{I I}, \mathrm{Co}^{\mathrm{II}}\right.$, $\mathrm{Ni}^{I I}, \mathrm{Zn}{ }^{I I}$ or $\mathrm{Cd} d^{I I} \mathrm{X}=\mathrm{Cl}^{-}$or $\mathrm{Br}^{-}$and $\mathrm{H}_{2} L=L-1$ or $\left.L-2\right)$. About 0.01 mole of metal halide was dissolved in $40-50 \mathrm{~mL}$ ethanol and treated with 0.01 mole of appropriate ligand in dry ethanol $(30-40 \mathrm{~mL})$ slowly with constant stirring. The resulting solution was refluxed on a steam bath for half an hour and concentrated to $20-30 \mathrm{~mL}$. The concentrated solution was chilled when crystalline precipitate separated. In case of $\mathrm{Cu}$ (II) the product were obtained immediately. The product was filtered, washed with cold dry ethanol, and dried over $\mathrm{CaCl}_{2}$. Yield $80-85 \%$.

2.2. Preparation of Bis Ligated Complexes $\left[\mathrm{M}(\mathrm{HL})_{2}\right], n \mathrm{H}_{2} \mathrm{O}$ $\left(\mathrm{M}=\mathrm{Mn}{ }^{I I}, \mathrm{Fe}^{I I}, \mathrm{Co}^{I I}, \mathrm{Ni}^{\mathrm{II}}, \mathrm{Cu}^{\mathrm{II}}, \mathrm{Zn}^{\mathrm{II}}, \mathrm{Cd}^{I I}\right.$ or $\mathrm{Pd}{ }^{I I}, \mathrm{H}_{2} \mathrm{~L}=\mathrm{H}_{2} \mathrm{~L}$ 1 or $\mathrm{H}_{2} \mathrm{~L}-2$ and $n=0$ but 2 for $\mathrm{Ni}^{I I}$ or $\left.\mathrm{Co}^{I I}\right)$. About 20 millimoles of ligand was dissolved in $80-90 \mathrm{~mL}$ hot methanol and treated slowly with aqueous methanolic solution of 10 millimoles of appropriate metal acetate \{chloride in case of $\mathrm{Pd}(\mathrm{II})$ and sulphate in case of $\mathrm{Fe}(\mathrm{II})\}$ and refluxed on steam bath for half an hour. In some cases a little aqueous solution of sodium acetate was added to adjust the $\mathrm{pH}$ 6-8. In most of the cases complexes were obtained immediately on diluting them with water. The products were digested on a steam bath and collected on a filter, washed with aqueous methanol, and dried over $\mathrm{CaCl}_{2}$. Yield is $95-98 \%$.

2.3. Preparation of $[M L B] \cdot n B\left(M=C u^{I I}, P d^{I I}, N i^{I I}, Z n^{I I}\right.$ or $\mathrm{Cd}^{I I} B=\mathrm{H}_{2} \mathrm{O}$, py or $Y$-pic and $n=0, \mathrm{H}_{2} \mathrm{~L}=\mathrm{H}_{2} \mathrm{~L}-1$ or $\mathrm{H}_{2} \mathrm{~L}-2$, if $\mathrm{B}=\mathrm{H}_{2} \mathrm{O}, n=1$ for $\mathrm{Ni}^{\mathrm{II}}$ and $\mathrm{H}_{2} \mathrm{~L}-1$ or $\mathrm{H}_{2} \mathrm{~L}-2$ ). About 5 or 10 millimole of metal chloride was dissolved in $30-40 \mathrm{~mL}$ aqueous methanol and treated with 5-6 mL pyridine or $Y$ pic. The resulting solution was treated with 5 or 10 millimole of ligand dissolved in hot methanol $(20-25 \mathrm{~mL})$.The $\mathrm{pH}$ of the solution was raised by adding dilute ammonia when appropriate complexes separated. The aqua complexes were obtained on refluxing [MLPy] in hot water in presence of a little ammonia. The products were collected on a filter, washed with cold aqueous methanol, and dried over $\mathrm{CaCl}_{2}$. The results of elemental analysis and physical data of complexes are presented in Tables 1 and 2. 
TABLE 1: Analytical results and physical data of complexes.

\begin{tabular}{|c|c|c|c|c|c|c|}
\hline \multirow{2}{*}{ Compound } & \multirow{2}{*}{ Colour } & \multicolumn{3}{|c|}{$\%$ analysis found (Calc) } & \multirow{2}{*}{$\mu_{\mathrm{eff}}$ B.M $\left(305^{\circ} \mathrm{K}\right)$} & \multirow{2}{*}{$\Omega \alpha \mathrm{mol}^{-1} \mathrm{ohm}^{-1} \mathrm{~cm}^{2}, 30^{\circ} \mathrm{C}$} \\
\hline & & $\mathrm{M}$ & $\mathrm{N}$ & Halogen & & \\
\hline $\mathrm{Cu}\left(\mathrm{H}_{2} \mathrm{~L}-1\right) \mathrm{Cl}_{2}$ & Green ash & $14.31(14.52)$ & $15.73(16.00)$ & $16.01(16.25)$ & 1.81 & 12 \\
\hline $\mathrm{Cu}\left(\mathrm{H}_{2} \mathrm{~L}-1\right) \mathrm{Br}_{2}$ & Brown & $11.91(12.07)$ & $13.31(13.48)$ & $30.11(30.44)$ & 1.84 & 8 \\
\hline $\mathrm{Zn}\left(\mathrm{H}_{2} \mathrm{~L}-1\right) \mathrm{Cl}_{2}$ & White & $14.61(14.88)$ & $15.91(16.18)$ & $15.01(15.12)$ & Dia & 10 \\
\hline $\mathrm{Zn}\left(\mathrm{H}_{2} \mathrm{~L}-1\right) \mathrm{Br}_{2}$ & White & $12.31(12.52)$ & $13.01(13.25)$ & $30.11(30.25)$ & Dia & 8 \\
\hline $\mathrm{Cd}\left(\mathrm{H}_{2} \mathrm{~L}-1\right) \mathrm{Cl}_{2}$ & Cream & $22.86(23.13)$ & $14.11(14.29)$ & $14.36(14.53)$ & Dia & 11 \\
\hline $\mathrm{Cd}\left(\mathrm{H}_{2} \mathrm{~L}-1\right) \mathrm{Br}_{2}$ & Cream & $19.46(19.54)$ & $12.11(12.32)$ & $27.38(27.67)$ & Dia & 10 \\
\hline $\mathrm{Ni}\left(\mathrm{H}_{2} \mathrm{~L}-1\right) \mathrm{Cl}_{2}$ & Greenish yellow & $13.32(13.54)$ & $16.31(16.48)$ & $16.57(16.70)$ & 3.18 & 10 \\
\hline $\mathrm{Co}\left(\mathrm{H}_{2} \mathrm{~L}-1\right) \mathrm{Cl}_{2}$ & Brown & $13.41(13.58)$ & $16.28(16.49)$ & $16.51(16.71)$ & 3.25 & 11 \\
\hline $\mathrm{Ni}\left(\mathrm{H}_{2} \mathrm{~L}-1\right) \mathrm{Br}_{2}$ & Bottle green & $11.10(11.21)$ & $13.50(13.61)$ & $30.17(30.64)$ & 3.21 & 8 \\
\hline $\mathrm{Cu}\left(\mathrm{H}_{2} \mathrm{~L}-2\right) \mathrm{Cl}_{2}$ & Ash green & $12.53(12.71)$ & $14.21(14.02)$ & $13.87(14.19)$ & 1.81 & 11 \\
\hline $\mathrm{Cu}\left(\mathrm{H}_{2} \mathrm{~L}-2\right) \mathrm{Br}_{2}$ & Brown & $10.61(10.79)$ & $11.63(11.81)$ & $26.93(27.13)$ & 1.83 & 8 \\
\hline $\mathrm{Cd}\left(\mathrm{H}_{2} \mathrm{~L}-2\right) \mathrm{Cl}_{2}$ & Cream & $20.49(21.67)$ & $12.76(13.54)$ & $12.69(12.93)$ & Dia & 11 \\
\hline $\mathrm{Zn}\left(\mathrm{H}_{2} \mathrm{~L}-2\right) \mathrm{Cl}_{2}$ & Cream & $13.31(13.45)$ & $13.21(13.96)$ & $14.00(14.15)$ & Dia & 10 \\
\hline $\mathrm{Cd}\left(\mathrm{H}_{2} \mathrm{~L}-2\right) \mathrm{Br}_{2}$ & Cream & $17.38(17.64)$ & $10.71(10.98)$ & $23.01(23.30)$ & Dia & 9 \\
\hline $\mathrm{Zn}\left(\mathrm{H}_{2} \mathrm{~L}-2\right) \mathrm{Br}_{2}$ & Cream & $10.91(11.08)$ & $11.61(11.84)$ & $26.81(27.06)$ & Dia & 11 \\
\hline $\mathrm{Ni}\left(\mathrm{H}_{2} \mathrm{~L}-2\right) \mathrm{Cl}_{2}$ & Greenish yellow & $11.61(11.86)$ & $13.96(14.15)$ & $14.16(14.35)$ & 3.20 & 12 \\
\hline $\mathrm{Co}\left(\mathrm{H}_{2} \mathrm{~L}-2\right) \mathrm{Cl}_{2}$ & Brown & 11.68 (11.89) & $13.89(14.16)$ & $14.23(14.36)$ & 3.32 & 13 \\
\hline $\mathrm{Ni}\left(\mathrm{H}_{2} \mathrm{~L}-2\right) \mathrm{Br}_{2}$ & Bottle green & $9.92(10.05)$ & $11.71(11.99)$ & $27.01(27.38)$ & 3.18 & 14 \\
\hline
\end{tabular}

$\left(\mathrm{H}_{2} \mathrm{~L}-1\right)=\mathrm{H}_{2}$ bmctz and $\left(\mathrm{H}_{2} \mathrm{~L}-2\right)=\mathrm{H}_{2}$ bpmctz.

\section{Results and Discussion}

The ligands N-[(1E)-1-(1H-benzimidazol-2-yl)ethylidene] morpholine-4-carbothiohydrazide $\left(\mathrm{H}_{2} \mathrm{~L}-1\right)$ and $\mathrm{N}-[(1 \mathrm{E})-1-$ (1H-benzimidazol-2-yl)phenyl(methylidene) ] morpholine4-carbothiohydrazide $\left(\mathrm{H}_{2} \mathrm{~L}-2\right)$ are potential $\mathrm{N} \mathrm{N} \mathrm{S}$ donor coordinating molecule capable of forming five membered strong chelates with The elemental analysis of complexes of $\mathrm{Ni}^{\mathrm{II}}, \mathrm{Co}^{\mathrm{II}}, \mathrm{Cu}^{\mathrm{II}}, \mathrm{Zn}^{\mathrm{II}}, \mathrm{Cd}^{\mathrm{II}}$ and halide formed in dry ethanol corresponds to compositions $\left[\mathrm{M}\left(\mathrm{H}_{2} \mathrm{~L}\right) \mathrm{X}_{2}\right]$, and $\left(\mathrm{H}_{2} \mathrm{~L}=\mathrm{H}_{2} \mathrm{~L}-1\right.$ or $\mathrm{H}_{2} \mathrm{~L}-2, \mathrm{X}=\mathrm{Cl}$ or $\mathrm{Br}$ ) metal ions. Depending on $\mathrm{pH}$ of medium, these ligands coordinate as neutral, monoanionic, and dianionic donor molecules. The elemental analysis of complexes of $\mathrm{Mn}^{\mathrm{II}}, \mathrm{Fe}^{\mathrm{II}}, \mathrm{Ni}^{\mathrm{II}}, \mathrm{Co}^{\mathrm{II}}, \mathrm{Cu}^{\mathrm{II}}, \mathrm{Zn}^{\mathrm{II}}, \mathrm{Cd}^{\mathrm{II}}$, and $\mathrm{Pd}^{\mathrm{II}}$ corresponds to compositions $\left[\mathrm{M}\left(\mathrm{H}_{2} \mathrm{~L}\right) \mathrm{X}_{2}\right]\left(\mathrm{M}=\mathrm{Cu}^{\mathrm{II}}, \mathrm{Ni}^{\mathrm{II}}\right.$, $\mathrm{Zn}^{\mathrm{II}}$ or $\mathrm{Cd}^{\mathrm{II}}$ and $\mathrm{X}=\mathrm{Cl}^{-}$or $\left.\mathrm{Br}^{-}\right)\left[\mathrm{M}(\mathrm{HL})_{2}\right] n \mathrm{H}_{2} \mathrm{O}\left(\mathrm{M}=\mathrm{Mn}^{\mathrm{II}}\right.$, $\mathrm{Fe}^{\mathrm{II}}, \mathrm{Co}^{\mathrm{II}}, \mathrm{Ni}^{\mathrm{II}}, \mathrm{Cu}^{\mathrm{II}}, \mathrm{Zn}^{\mathrm{II}}, \mathrm{Cd}^{\mathrm{II}}$ or $\mathrm{Pd}^{\mathrm{II}}, \mathrm{H}_{2} \mathrm{~L}=\mathrm{H}_{2} \mathrm{~L}-1$ or $\mathrm{H}_{2} \mathrm{~L}-2$, $n=0$ but 2 for $\mathrm{Co}^{\mathrm{II}}$ and $\left.\mathrm{Ni}^{\mathrm{II}}\right)$ and $[\mathrm{MLB}] n \mathrm{~B}\left(\mathrm{M}=\mathrm{Ni}^{\mathrm{II}}, \mathrm{Cu}^{\mathrm{II}}\right.$, $\mathrm{Pd}^{\mathrm{II}}, \mathrm{Zn}^{\mathrm{II}}$ or $\mathrm{Cd}^{\mathrm{II}}$ and $\mathrm{H}_{2} \mathrm{~L}=\mathrm{H}_{2} \mathrm{~L}-1$ or $\mathrm{H}_{2} \mathrm{~L}-2, \mathrm{~B}=\mathrm{Py}, \mathrm{Y}$-pic or $\mathrm{H}_{2} \mathrm{O}$ and $n=0$ or 1 ). These complexes are quite stable at room temperature and do not lose texture in desiccator. On heating the complexes $\left[\mathrm{M}(\mathrm{HL})_{2} \cdot n \mathrm{H}_{2} \mathrm{O}\left(\mathrm{M}=\mathrm{Co}^{\mathrm{II}}\right.\right.$, or $\mathrm{Ni}^{\mathrm{II}}$, and $\mathrm{HL}=\mathrm{HL}-1$ or HL-2) in air oven between 60 and $80^{\circ} \mathrm{C}$ for one hour, is loss $\mathrm{H}_{2} \mathrm{O}$ and loss in weight incurred in complexes correspond to loss of required for $2 \mathrm{H}_{2} \mathrm{O}$, and no change in colour of the complexes that was noticed which indicated that $\mathrm{H}_{2} \mathrm{O}$ molecules are not coordinated to metal atom rather they are held up in crystal lattices. The weight loss incurred in complexes $\mathrm{Ni}(\mathrm{L}-1) 2 \mathrm{H}_{2} \mathrm{O}$ and $\mathrm{Ni}(\mathrm{L}-2) 2 \mathrm{H}_{2} \mathrm{O}$ at $120^{\circ} \mathrm{C}$ corresponds to loss of only one $\mathrm{H}_{2} \mathrm{O}$, indicating that one $\mathrm{H}_{2} \mathrm{O}$ is coordinated to nickel (II) in complex [ $\left.\mathrm{NiL}\left(\mathrm{H}_{2} \mathrm{O}\right)\right] \mathrm{H}_{2} \mathrm{O}$ and the second $\mathrm{H}_{2} \mathrm{O}$ is held up in crystal lattices. The dihalo complexes are formed in dry ethanol by interacting solutions of ligand with metal halides. The bis ligated complexes $\left[\mathrm{M}(\mathrm{HL})_{2}\right] n \mathrm{H}_{2} \mathrm{O}$ were formed in neutral or weakly basic medium ( $\mathrm{pH}$ 6-8) by interacting aqueous methanolic or ethanolic solutions of metal acetate or metal chloride and appropriate ligand in 1:2 molar proportion. The ligands coordinate as dianionic tridentate molecule in much basic medium ( $\mathrm{pH}$ 9-12) and form complexes of stoichiometry, $[\mathrm{MLB}] \cdot n \mathrm{~B}\left(\mathrm{M}=\mathrm{Cu}^{\mathrm{II}}, \mathrm{Ni}^{\mathrm{II}}, \mathrm{Zn}^{\mathrm{II}}, \mathrm{Cd}^{\mathrm{II}}\right.$ or $\mathrm{Pd}^{\mathrm{II}}$ and $\mathrm{H}_{2} \mathrm{~L}=\mathrm{H}_{2} \mathrm{~L}-1$ or $\mathrm{H}_{2} \mathrm{~L}-2, \mathrm{~B}=\mathrm{H}_{2} \mathrm{O}$, py or $Y$-pic and $n=0$ or 1 ). The complexes are all insoluble in water but aqueous ethanolic suspension of $\left[\mathrm{M}\left(\mathrm{H}_{2} \mathrm{~L}\right) \mathrm{X}_{2}\right]$ gradually dissociates into $\mathrm{M}(\mathrm{HL})_{2}$ and $\mathrm{MX}_{2}$ giving conducting solution. Freshly prepared DMF solution of complexes, however shows negligible electric conductance value indicating them to be nonionic and even halogens are coordinated to metal atom [36]. Zinc(II), cadmium(II), and nickel(II) complexes $[\mathrm{NiLB}] \cdot n \mathrm{~B}$ are diamagnetic. At room temperature all copper(II) complexes show normal magnetic moment value (1.81-1.89 B.M) and magnetic moment value of $\mathrm{Mn}(\mathrm{II})$ complexes $\left[\mathrm{Mn}(\mathrm{HL})_{2}\right]$ (5.88-5.92 B.M), $\mathrm{Ni}(\mathrm{II})$ complexes $\left[\mathrm{Ni}(\mathrm{HL})_{2}\right](2.93-3.01 \mathrm{~B} . \mathrm{M})$, and $\mathrm{Fe}(\mathrm{II})$ complexes $\mathrm{Fe}(\mathrm{HL})_{2}$ (4.89-4.91 B.M) corresponds to spin free octahedral geometry of metal ions [37]. The moment value of cobalt(II) complexes $\left[\mathrm{Co}(\mathrm{HL})_{2}\right](3.26-3.68$ B.M) is lower than spin free octahedral geometry and anomalous probably due to spin-exchange interactions [38]. The dihalo complexes $\left[\mathrm{Co}(\mathrm{HL}) \mathrm{X}_{2}\right]$ show magnetic moment value (4.54-4.71 B.M) similar to five coordinated TBP structure $[37,38]$. As expected zinc(II) or Cd(II) complexes $\mathrm{M}(\mathrm{HL})_{2}$ and $\left[\mathrm{M}\left(\mathrm{H}_{2} \mathrm{~L}\right) \mathrm{X}_{2}\right]\left(\mathrm{X}=\mathrm{Cl}^{-}\right.$or $\left.\mathrm{Br}^{-}\right)$are diamagnetic. The electronic absorption spectra of ligand $\left(\mathrm{H}_{2} \mathrm{~L}-1\right.$ or $\left.\mathrm{H}_{2} \mathrm{~L}-2\right)$ and their complexes were determined in ethanol or ethanol 
TABLE 2: Analytical results and physical data of complexes.

\begin{tabular}{|c|c|c|c|c|c|c|}
\hline \multirow{2}{*}{$\begin{array}{l}\text { Compound } \\
\mathrm{H}_{2} \mathrm{~L}=\mathrm{H}_{2} \text { bmctz } \\
\mathrm{H}_{2} \mathrm{~L}^{\prime}=\mathrm{H}_{2} \text { bpmctz }\end{array}$} & \multirow{2}{*}{ Colour } & \multicolumn{3}{|c|}{$\%$ analysis found $/($ Calc $)$} & \multirow{2}{*}{$\mu_{\mathrm{eff}}$ B.M $305^{\circ} \mathrm{K}$} & \multirow{2}{*}{$\wedge \alpha \mathrm{mol}^{-1} \mathrm{Ohm}^{-1} \mathrm{~cm}^{2}, 30^{\circ} \mathrm{C}$} \\
\hline & & M & $\mathrm{N}$ & Sulphur & & \\
\hline$\left[\mathrm{Co}(\mathrm{HL}-1)_{2}\right] 2 \mathrm{H}_{2} \mathrm{O}$ & Brown & $8.61(8.88)$ & $21.01(21.12)$ & $9.50(9.65)$ & 3.26 & 8 \\
\hline$\left[\mathrm{Cu}(\mathrm{HL}-1)_{2}\right]$ & Ash colour & $9.31(9.52)$ & $20.87(21.09)$ & $9.31(9.59)$ & 1.86 & 7 \\
\hline$\left[\mathrm{Mn}(\mathrm{HL}-1)_{2}\right]$ & Dull cream & $8.14(8.33)$ & $21.22(21.24)$ & $9.41(9.78)$ & 5.88 & 8 \\
\hline$\left[\mathrm{Fe}(\mathrm{HL}-1)_{2}\right]$ & Light brown & $8.31(8.47)$ & $21.01(21.20)$ & $9.48(9.64)$ & 4.91 & 6 \\
\hline$\left[\mathrm{Cu}(\mathrm{HL}-2)_{2}\right]$ & Ash green & $7.91(8.01)$ & $17.61(17.79)$ & $8.00(8.08)$ & 1.88 & 8 \\
\hline$\left[\mathrm{Mn}(\mathrm{HL}-2)_{2}\right]$ & Dull cream & $6.88(7.01)$ & $17.61(17.88)$ & $9.11(9.26)$ & 5.92 & 8 \\
\hline$\left[\mathrm{Fe}(\mathrm{HL}-2)_{2}\right]$ & Light brown & $7.01(7.12)$ & $17.15(17.86)$ & $9.11(9.24)$ & 4.86 & 6 \\
\hline$\left[\mathrm{Co}(\mathrm{HL}-2)_{2}\right] 2 \mathrm{H}_{2} \mathrm{O}$ & Brown & $7.02(7.16)$ & $17.00(17.01)$ & $7.61(7.77)$ & 3.68 & 10 \\
\hline$\left[\mathrm{Ni}(\mathrm{HL}-1)_{2}\right] 2 \mathrm{H}_{2} \mathrm{O}$ & Reddish brown & $8.37(8.40)$ & $19.81(20.02)$ & $9.00(9.22)$ & 3.13 & 8 \\
\hline$\left[\mathrm{Ni}(\mathrm{HL}-2)_{2}\right] 2 \mathrm{H}_{2} \mathrm{O}$ & Reddish brown & $7.10(7.13)$ & $16.81(17.01)$ & $7.57(7.78)$ & 2.98 & 7 \\
\hline$\left[\mathrm{Zn}(\mathrm{HL}-1)_{2}\right]$ & Yellow & $9.82(9.76)$ & $20.73(20.90)$ & $9.09(9.10)$ & Dia & 10 \\
\hline$\left[\mathrm{Zn}(\mathrm{HL}-2)_{2}\right]$ & Yellow & $8.01(8.29)$ & $17.51(17.65)$ & $8.01(8.06)$ & Dia & 8 \\
\hline$\left[\mathrm{Pd}(\mathrm{HL}-1)_{2}\right]$ & Reddish brown & $14.61(14.98)$ & $19.56(19.71)$ & $8.90(9.01)$ & Dia & 9 \\
\hline$\left[\mathrm{Pd}(\mathrm{HL}-2)_{2}\right]$ & Reddish brown & $12.61(12.75)$ & $16.66(16.87)$ & $7.51(7.67)$ & Dia & 7 \\
\hline$[\mathrm{Pd}(\mathrm{L}-1) \mathrm{Py}]$ & Orange & $21.63(21.75)$ & $17.01(17.27)$ & $6.51(6.58)$ & Dia & 6 \\
\hline$[\mathrm{Pd}(\mathrm{L}-2) \mathrm{Py}]$ & Orange & $19.31(19.40)$ & $15.01(15.32)$ & $5.69(5.83)$ & Dia & 8 \\
\hline$[\mathrm{Pd}(\mathrm{L}-1)(Y-\mathrm{pic})]$ & Orange & $21.11(21.27)$ & $16.68(16.86)$ & $6.30(6.39)$ & Dia & 8 \\
\hline$[\mathrm{Pd}(\mathrm{L}-2)(Y$-pic $)]$ & Orange & $18.68(18.98)$ & $14.91(15.01)$ & $5.68(5.73)$ & Dia & 7 \\
\hline$[\mathrm{Ni}(\mathrm{L}-1) \mathrm{Py}]$ & Yellowish red & $13.31(13.38)$ & $19.11(19.21)$ & $7.10(7.29)$ & Dia & 8 \\
\hline$[\mathrm{Ni}(\mathrm{L}-1)(Y$-pic $)]$ & Yellowish red & $12.78(12.99)$ & $18.48(18.59)$ & $6.87(7.08)$ & Dia & 7 \\
\hline$\left[\mathrm{Ni}(\mathrm{L}-1)\left(\mathrm{H}_{2} \mathrm{O}\right)\right] \mathrm{H}_{2} \mathrm{O}$ & Yellowish orange & $14.63(14.83)$ & $17.51(17.69)$ & $8.11(8.06)$ & Dia & 9 \\
\hline$[\mathrm{Ni}(\mathrm{L}-2) \mathrm{Py}]$ & Orange yellow & $11.43(11.72)$ & $16.71(16.77)$ & $6.11(6.39)$ & Dia & 10 \\
\hline$[\mathrm{Ni}(\mathrm{L}-2)(Y$-pic $)]$ & Orange yellow & $11.14(11.42)$ & $16.21(16.32)$ & $6.01(6.23)$ & Dia & 8 \\
\hline$\left[\mathrm{Ni}(\mathrm{L}-2)\left(\mathrm{H}_{2} \mathrm{O}\right)\right] \mathrm{H}_{2} \mathrm{O}$ & Orange yellow & $12.78(12.82)$ & $15.11(15.29)$ & $6.81(6.99)$ & Dia & 7 \\
\hline$[\mathrm{Cd}(\mathrm{L}-1)(Y$-pic $)]$ & Orange yellow & $22.01(22.22)$ & $16.51(16.62)$ & $6.11(6.33)$ & Dia & 10 \\
\hline$\left[\mathrm{Cd}(\mathrm{L}-1)\left(\mathrm{H}_{2} \mathrm{O}\right)\right]$ & Orange yellow & $26.15(26.05)$ & $16.02(16.23)$ & $7.31(7.42)$ & Dia & 8 \\
\hline [Cd(L-1)Py] & Orange yellow & $22.61(22.81)$ & $17.34(17.51)$ & $6.41(6.49)$ & Dia & 9 \\
\hline [Cd(L-2)Py] & Orange yellow & $20.11(20.21)$ & $15.01(15.15)$ & $5.70(5.77)$ & Dia & 11 \\
\hline$[\mathrm{Cd}(\mathrm{L}-2)(Y-\mathrm{pic})]$ & Orange yellow & $19.61(19.88)$ & $14.92(14.88)$ & $5.41(5.64)$ & Dia & 10 \\
\hline$\left[\mathrm{Cd}(\mathrm{L}-2)\left(\mathrm{H}_{2} \mathrm{O}\right)\right]$ & Yellow & $22.48(22.78)$ & $14.21(14.19)$ & $6.51(6.48)$ & Dia & 9 \\
\hline$[\mathrm{Zn}(\mathrm{L}-2) \mathrm{Py}]$ & Orange yellow & $14.51(14.62)$ & $18.76(18.86)$ & $7.00(7.18)$ & Dia & 10 \\
\hline$\left[\mathrm{Zn}(\mathrm{L}-1)\left(\mathrm{H}_{2} \mathrm{O}\right)\right]$ & Yellow & $17.14(17.01)$ & $18.41(18.23)$ & $8.10(8.32)$ & Dia & 8 \\
\hline$[\mathrm{Zn}(\mathrm{L}-2) \mathrm{Py}]$ & Yellow & $12.81(12.88)$ & $16.47(16.55)$ & $6.03(6.36)$ & Dia & 6 \\
\hline$[\mathrm{Zn}(\mathrm{L}-2)(Y$-pic $)]$ & Orange yellow & $12.41(12.54)$ & $15.91(16.11)$ & $5.91(6.14)$ & Dia & 9 \\
\hline$\left[\mathrm{Zn}(\mathrm{L}-2)\left(\mathrm{H}_{2} \mathrm{O}\right)\right]$ & Yellow & $14.61(14.72)$ & $15.58(15.78)$ & $6.91(7.17)$ & Dia & 8 \\
\hline$[\mathrm{Zn}(\mathrm{L}-1)(Y$-pic $)]$ & Yellow & $14.21(14.23)$ & $18.41(18.59)$ & $6.91(7.08)$ & Dia & 11 \\
\hline$[\mathrm{Cu}(\mathrm{L}-1) \mathrm{Py}]$ & Bottle green & $14.01(14.32)$ & $18.32(18.54)$ & $7.03(7.21)$ & 1.86 & 8 \\
\hline$[\mathrm{Cu}(\mathrm{L}-2) \mathrm{Py}]$ & Bottle green & $13.81(13.95)$ & $18.71(18.93)$ & $7.11(7.21)$ & 1.84 & 9 \\
\hline$[\mathrm{Cu}(\mathrm{L}-1)(Y-\mathrm{pic})]$ & Ash colour & $13.61(13.88)$ & $18.17(18.36)$ & $6.81(6.99)$ & 1.89 & 6 \\
\hline$[\mathrm{Cu}(\mathrm{L}-2)(Y-\mathrm{pic})]$ & Ash colour & $12.31(12.25)$ & $16.01(16.20)$ & $6.01(6.16)$ & 1.84 & 5 \\
\hline$\left[\mathrm{Cu}(\mathrm{L}-2)\left(\mathrm{H}_{2} \mathrm{O}\right)\right]$ & Brown & $13.61(13.73)$ & $15.00(15.14)$ & $6.71(6.91)$ & 1.79 & 8 \\
\hline$\left[\mathrm{Cu}(\mathrm{L}-1)\left(\mathrm{H}_{2} \mathrm{O}\right)\right]$ & Brown & $15.48(15.74)$ & $17.13(17.35)$ & $7.76(7.93)$ & 1.78 & 10 \\
\hline
\end{tabular}


TABLE 3: Prominent I.R. bands of ligands and their complexes in $\mathrm{cm}^{-1}$.

\begin{tabular}{|c|c|c|c|c|c|}
\hline Compound & $\begin{array}{c}v(\mathrm{NH}), v(\mathrm{OH}), v\left(\mathrm{CH}_{2}\right) \\
+v(\mathrm{C}-\mathrm{H})\end{array}$ & $\begin{array}{c}v(\mathrm{C}=\mathrm{N}) \text { imidazole } \\
\nu(\mathrm{C}=\mathrm{N}) \text { azomethene }\end{array}$ & Ring breathing mode & $\nu(\mathrm{C}=\mathrm{S})$ & $\begin{array}{l}\nu(\mathrm{M}-\mathrm{N}) \\
\nu(\mathrm{M}-\mathrm{S})\end{array}$ \\
\hline $\mathrm{H}_{2} \mathrm{~L}-1$ & $\begin{array}{c}3350,3240,3120,3040 \\
2949,2860\end{array}$ & $1653,1610,1585$ & 1040 & 912 & 435,415 \\
\hline $\mathrm{H}_{2} \mathrm{~L}-2$ & $3238,3190,3060,2950,2832$ & $1628,1605,1585$ & 1035 & 938 & 431,418 \\
\hline$\left[\mathrm{Cu}\left(\mathrm{H}_{2} \mathrm{~L}-1\right) \mathrm{Cl}_{2}\right]$ & $\begin{array}{c}3280,3235,3125,3040 \\
2950,2850\end{array}$ & $1622,1590,1580$ & 1055 & 892 & 427 \\
\hline$\left[\mathrm{Zn}\left(\mathrm{H}_{2} \mathrm{~L}-1\right) \mathrm{Cl}_{2}\right]$ & $\begin{array}{c}3320,3235,3140,3035 \\
2960,2845\end{array}$ & $1615,1595,1580$ & 1024 & 896 & 429 \\
\hline$\left[\mathrm{Cu}\left(\mathrm{H}_{2} \mathrm{~L}-2\right) \mathrm{Cl}_{2}\right]$ & $\begin{array}{c}3340,3230,3145,3045 \\
2960,2840\end{array}$ & $1621,1610,1590$ & 1025 & 908 & 436,413 \\
\hline$\left[\mathrm{Ni}\left(\mathrm{H}_{2} \mathrm{~L}-2\right) \mathrm{Cl}_{2}\right]$ & $\begin{array}{c}3340,3240,3140,3045 \\
2950,2840\end{array}$ & $1618,1595,1584$ & 1040 & 912 & 428,410 \\
\hline$\left[\mathrm{Co}(\mathrm{HL}-1)_{2}\right] 2 \mathrm{H}_{2} \mathrm{O}$ & $\begin{array}{c}3320,3245,3140,3050, \\
2960,2840\end{array}$ & $1618,1602,1595$ & 1035 & 768 & 441,418 \\
\hline$\left[\mathrm{Zn}(\mathrm{HL}-2)_{2}\right]$ & $\begin{array}{c}3340-3140,3050,2960 \\
2850\end{array}$ & $1622,1605,1592$ & 1030 & 772 & 438,416 \\
\hline$\left[\mathrm{Ni}(\mathrm{HL}-2)_{2}\right] 2 \mathrm{H}_{2} \mathrm{O}$ & 3380-3100br, 2960, 2840 & $1615,1600,1595$ & 1040 & 782 & 435,418 \\
\hline$\left[\mathrm{Cd}(\mathrm{HL}-2)_{2}\right]$ & $3320,3240,3130,2960,2850$ & $1612,1595,1580$ & 1038 & 788 & 436,417 \\
\hline$\left[\mathrm{Co}(\mathrm{HL}-2)_{2}\right] 2 \mathrm{H}_{2} \mathrm{O}$ & 3400-3120br, 2960, 2840 & $1618,1605,1585$ & 1028 & 792 & 438,422 \\
\hline$[\mathrm{Ni}(\mathrm{L}-1) \mathrm{Py}]$ & $\begin{array}{c}3320-3180,3040,2980 \\
2845\end{array}$ & $1612,1595,1575$ & 1024 & 760 & 443,418 \\
\hline$[\mathrm{Cu}(\mathrm{L}-2)] Y$-pic & $\begin{array}{c}3340,3260,3140,3050, \\
2840\end{array}$ & $1620,1590,1580$ & 1025 & 772 & 434,415 \\
\hline$[\mathrm{Cu}(\mathrm{L}-2)] 2 \mathrm{H}_{2} \mathrm{O}$ & $3350-3060,2950,2840$ & $1620,1600,1580$ & 1025 & 781 & 438,410 \\
\hline$[\mathrm{Zn}(\mathrm{L}-1) \mathrm{Py}]$ & $\begin{array}{c}3320,3270,3130,3040 \\
2955,2845\end{array}$ & 1615,16001590 & 1030 & 776 & 436,413 \\
\hline
\end{tabular}

DMF (50\%) mixed solvent in the range $200-850 \mathrm{~nm}$. The ligand $\mathrm{H}_{2} \mathrm{~L}-1$ displays strong bands at 214, 252, and 268 and a medium band at $295 \mathrm{~nm}$ attributed from $\sigma \rightarrow \sigma^{*}$, $\sigma \rightarrow \pi^{*}, \pi \rightarrow \pi^{*}$, and $n \rightarrow \pi^{*}$ transitions of ligand molecule [39]. The electronic absorption bands of $\mathrm{H}_{2} \mathrm{~L}-2$ are observed as strong band at 212, 245, 270, and $290 \mathrm{~nm}$ attributed from $\sigma \rightarrow \sigma^{*}, \sigma \rightarrow \pi^{*}, \pi \rightarrow \pi^{*}$, and $n \rightarrow \pi^{*}$ $(\mathrm{C}=\mathrm{S})$ of ligand. In complexes these transition are mostly enveloped by strong charge transfer from $\mathrm{M} \rightarrow \mathrm{L}$ and $\mathrm{L} \rightarrow \mathrm{M}$ electronic transitions. The metal complexes form very deep coloured solutions in DMF-ethanol mixture and distinct d-d electronic transitions could not be located below 380-390 nm. The $\mathrm{Zn}(\mathrm{II})$ and Cd(II) complexes as expected do not have $\mathrm{d}-\mathrm{d}$ electronic transition but due to charge transfer band show strong absorption near 420-400 nm. The medium band at $460-480 \mathrm{~nm}$ for [NiLB] $n \mathrm{~B}\left(\mathrm{H}_{2} \mathrm{~L}=\mathrm{H}_{2} \mathrm{~L}-1\right.$ or $\mathrm{H}_{2} \mathrm{~L}-2, \mathrm{~B}=$ py or $Y$-pic) is attributed to ${ }^{1} \mathrm{~A}_{1 \mathrm{~g}} \rightarrow{ }^{1} \mathrm{~B}_{1 \mathrm{~g}}$ transition in planar field [39]. In DMF-ethanol mixture the complexes $\left[\mathrm{Ni}(\mathrm{HL})_{2}\right]$ as well as $\left[\mathrm{Ni}\left(\mathrm{H}_{2} \mathrm{~L}\right) \mathrm{Cl}_{2}\right]$ have same colour and similar electronic absorption band near 460$480 \mathrm{~nm}$. The complexes probably acquire planar structure in basic DMF medium. The cobalt (II) complexes display weak electronic absorption near $430-440 \mathrm{~nm}$ and $560-580 \mathrm{~nm}$ assignable to ${ }^{4} \mathrm{~T}_{1 \mathrm{~g}} \rightarrow{ }^{4} \mathrm{~T}_{2 \mathrm{~g}}$ and ${ }^{4} \mathrm{~T}_{1 \mathrm{~g}} \rightarrow{ }^{4} \mathrm{~A}_{2 \mathrm{~g}},{ }^{4} \mathrm{~T}_{1 \mathrm{~g}}(\mathrm{P})$ transitions in octahedral field [39]. The $\mathrm{Cu}(\mathrm{II})$ complexes
$\left[\mathrm{Cu}(\mathrm{HL})_{2}\right]$ show strong absorption below 420 assignable to charge transfer transition. The complexes display $\mathrm{d}-$ $\mathrm{d}$ transitions at $450 \mathrm{~nm}$ (medium) and $650-680 \mathrm{~nm}$ as broad weak band assignable to ${ }^{2} \mathrm{~B}_{1 \mathrm{~g}} \rightarrow{ }^{2} \mathrm{~B}_{2 \mathrm{~g}},{ }^{2} \mathrm{E}_{\mathrm{g}}$, and ${ }^{2} \mathrm{~B}_{1 \mathrm{~g}} \rightarrow{ }^{2} \mathrm{~A}_{1 \mathrm{~g}}$ transition in distorted octahedral field [39]. In case of $\mathrm{Mn}$ (II) no distinct $\mathrm{d}-\mathrm{d}$ transitions could be located definitely, since all $\mathrm{d}-\mathrm{d}$ transitions of $\mathrm{Mn}$ (II) complexes are of spin forbidden type. Iron (II) complexes display a shoulder near 440 and $450 \mathrm{~nm}$ attributable to ${ }^{5} \mathrm{~T}_{2 \mathrm{~g}} \rightarrow{ }^{5} \mathrm{E}_{\mathrm{g}}$ transitions in octahedral field [37-39] (See Figures 1 and 2).

The IR spectra of ligands and their complexes were recorded as $\mathrm{KBr}$ optics in range $4000-400 \mathrm{~cm}^{-1}$. The diagnostic I.R. bands of ligands and some of their complexes are recorded in Table 3 . The ligand $\mathrm{H}_{2} \mathrm{~L}-1$ displays medium I.R. bands at 3350, 3240, 3120, 3040, 2949, 2860, and $2840 \mathrm{~cm}^{-1}$ attributed from $v(\mathrm{NH})$, phenyl $(\mathrm{C}-\mathrm{H})$ stretches, and morpholine ring $\nu\left(\mathrm{CH}_{2}\right)$ vibrations and most of them are retained in complexes. The $\nu(\mathrm{C}=\mathrm{N}), \nu(\mathrm{N}-\mathrm{N})$ of imidazole ring and azomethene part of ligand were observed at 1653, 1610 and $1585 \mathrm{~cm}^{-1}$. The $\delta(\mathrm{NH})$ of ligand was located at 1515 and $1485 \mathrm{~cm}^{-1}$. The IR spectrum of $\mathrm{Cu}\left(\mathrm{H}_{2} \mathrm{~L}-1\right) \mathrm{Cl}_{2}$ displays azomethene $\nu(\mathrm{C}=\mathrm{N})$ at $1622 \mathrm{~cm}^{-1}$ and imidazole ring $\nu(\mathrm{C}=\mathrm{N})$ at 1590 which is lower than free ligand molecule that indicated 
TABLE 4: Results of fungal activity (conc. of compound in ppm).

\begin{tabular}{|c|c|c|c|c|c|c|}
\hline Compound & Conc. in ppm & A. niger & A. flavus & F. oxisporum & R. bataticola & R.phaseoli \\
\hline \multirow{2}{*}{$\mathrm{H}_{2}$ bmctz $=\mathrm{H}_{2} \mathrm{~L}-1$} & 100 & 40 & 35 & 30 & 45 & 50 \\
\hline & 200 & 55 & 50 & 52 & 68 & 75 \\
\hline \multirow{2}{*}{$\mathrm{H}_{2} \mathrm{bpmctz}=\mathrm{H}_{2} \mathrm{~L}-2$} & 100 & 46 & 36 & 40 & 48 & 55 \\
\hline & 200 & 68 & 52 & 65 & 72 & 80 \\
\hline \multirow{2}{*}{$\mathrm{Cu}\left(\mathrm{H}_{2} \mathrm{~L}-1\right) \mathrm{Cl}_{2}$} & 100 & 48 & 55 & 32 & 50 & 55 \\
\hline & 200 & 65 & 70 & 55 & 75 & 85 \\
\hline \multirow{2}{*}{$\mathrm{Cu}\left(\mathrm{H}_{2} \mathrm{~L}-2\right) \mathrm{Cl}_{2}$} & 100 & 50 & 60 & 42 & 52 & 56 \\
\hline & 200 & 70 & 78 & 70 & 78 & 86 \\
\hline \multirow{2}{*}{$\mathrm{Cu}\left(\mathrm{H}_{2} \mathrm{~L}-1\right) \mathrm{Br}_{2}$} & 100 & 50 & 56 & 42 & 52 & 57 \\
\hline & 200 & 68 & 72 & 68 & 76 & 87 \\
\hline \multirow{2}{*}{$\mathrm{Cu}(\mathrm{HL}-1)_{2}$} & 100 & 48 & 50 & 35 & 50 & 54 \\
\hline & 200 & 62 & 65 & 60 & 70 & 80 \\
\hline \multirow{2}{*}{$\mathrm{Zn}\left(\mathrm{H}_{2} \mathrm{~L}-1\right)_{2} \mathrm{Cl}_{2}$} & 100 & 48 & 42 & 40 & 48 & 54 \\
\hline & 200 & 64 & 60 & 60 & 70 & 80 \\
\hline \multirow{2}{*}{$\mathrm{Zn}\left(\mathrm{H}_{2} \mathrm{~L}-2\right)_{2} \mathrm{Br}_{2}$} & 100 & 50 & 45 & 42 & 50 & 55 \\
\hline & 200 & 68 & 62 & 65 & 74 & 82 \\
\hline \multirow{2}{*}{$\mathrm{Zn}\left(\mathrm{H}_{2} \mathrm{~L}-2\right) \mathrm{Br}_{2}$} & 100 & 52 & 42 & 45 & 52 & 60 \\
\hline & 200 & 72 & 60 & 70 & 76 & 85 \\
\hline \multirow{2}{*}{$\mathrm{Mn}(\mathrm{HL}-1)_{2}$} & 100 & 42 & 40 & 35 & 46 & 50 \\
\hline & 200 & 58 & 53 & 55 & 70 & 75 \\
\hline \multirow{2}{*}{$\mathrm{Fe}(\mathrm{HL}-2)_{2}$} & 100 & 41 & 42 & 36 & 48 & 52 \\
\hline & 200 & 57 & 55 & 55 & 74 & 78 \\
\hline \multirow{2}{*}{$\mathrm{Pd}(\mathrm{L}-1) \mathrm{Py}$} & 100 & 52 & 45 & 40 & 50 & 52 \\
\hline & 200 & 68 & 68 & 62 & 72 & 78 \\
\hline \multirow{2}{*}{$\mathrm{Ni}\left(\mathrm{H}_{2} \mathrm{~L}-1\right) \mathrm{Cl}_{2}$} & 100 & 48 & 42 & 45 & 48 & 54 \\
\hline & 200 & 56 & 56 & 55 & 72 & 80 \\
\hline \multirow{2}{*}{$\mathrm{Ni}(\mathrm{L}-1) \mathrm{Py}$} & 100 & 46 & 40 & 32 & 45 & 52 \\
\hline & 200 & 56 & 52 & 55 & 70 & 76 \\
\hline \multirow{2}{*}{$\mathrm{Cd}(\mathrm{HL}-1)_{2}$} & 100 & 46 & 43 & 35 & 48 & 54 \\
\hline & 200 & 60 & 58 & 60 & 72 & 80 \\
\hline \multirow{2}{*}{$\mathrm{Cd}(\mathrm{HL}-2)_{2}$} & 100 & 48 & 42 & 43 & 50 & 60 \\
\hline & 200 & 66 & 60 & 65 & 74 & 85 \\
\hline \multirow{2}{*}{ Ref. Mancozeb } & 100 & 75 & 80 & 78 & 81 & 82 \\
\hline & 200 & 99 & 98 & 97 & 99 & 100 \\
\hline
\end{tabular}

the coordination of both $(\mathrm{C}=\mathrm{N})$ nitrogen to metal atom. The thione $v(\mathrm{C}=\mathrm{S})$ of ligand shifted from $912 \mathrm{~cm}^{-1}$ to $892 \mathrm{~cm}^{-1}$ in complex $\left[\mathrm{Cu}\left(\mathrm{H}_{2} \mathrm{~L}-1\right) \mathrm{Cl}_{2}\right]$ indicated the involvement of thiol sulphur in coordination. Thus the ligand is bonded as tridentate $\mathrm{N}, \mathrm{N}, \mathrm{S}$ donor molecule in complexes. The $\delta(\mathrm{NH})$ at 1515 and $\nu(\mathrm{N}-\mathrm{H})$ at $3240 \mathrm{~cm}^{-1}$ of ligand were absent in complexes with dianionic ligand $[\mathrm{M}(\mathrm{L}-1) \mathrm{B}]$. The complexes containing pyridine or $Y$-picoline molecule display a prominent band at $1590-1595$ and $1015-1025 \mathrm{~cm}^{-1}$ due to $\nu(\mathrm{C}=\mathrm{N})$ of coordinated pyridine ring and pyridine ring breathing mode of vibration [40]. The imidazole part $\nu(\mathrm{C}=\mathrm{N})$ and azomethene part $\nu(\mathrm{C}=\mathrm{N})$ stretches of ligand shift to lower frequency in complexes by $10-15 \mathrm{~cm}^{-1}$ that suggested the coordination of both $(\mathrm{CN})$ nitrogen. The thioamide $v(\mathrm{C}=\mathrm{S})$ of ligand was observed at $912 \mathrm{~cm}^{-1}$, which is shifted by $20-25 \mathrm{~cm}^{-1}$ in complexes of type $\left[\mathrm{M}\left(\mathrm{H}_{2} \mathrm{~L}-1\right) \mathrm{X}_{2}\right]$ indicating that $(\mathrm{C}=\mathrm{S})$ is bonded through thione sulphur. The $v(\mathrm{C}=\mathrm{S})$ of ligand shifts to $760-780 \mathrm{~cm}^{-1}$ in complexes $\left[\mathrm{M}(\mathrm{HL}-1)_{2}\right]$ and $[\mathrm{M}(\mathrm{L}-1) \mathrm{B}]$ type of complexes indicating the coordination of thioamide $(\mathrm{C}=\mathrm{S})$ through deprotonated thiol sulphur. The ligand $\mathrm{H}_{2} \mathrm{~L}-2$ shows $v(\mathrm{~N}-\mathrm{H}), v(\mathrm{C}-\mathrm{H})$ phenyl ring and $\left(\mathrm{CH}_{2}\right)$ ring vibration morpholine at 3238, 3190, 3060, 2950, and $2832 \mathrm{~cm}^{-1}$. These are retained with some change in intensity and band position in complexes. The complexes $\left[\mathrm{M}(\mathrm{HL})_{2}\right] 2 \mathrm{H}_{2} \mathrm{O},(\mathrm{HL}=\mathrm{HL}-2$ or HL-1) show broad IR band near $3180-3450 \mathrm{~cm}^{-1}$ attributed to hydrogen bonded $\nu(\mathrm{OH})$ of $\mathrm{H} 2 \mathrm{O}$. The $\nu(\mathrm{C}=\mathrm{N})$ of azomethene part and benzimidazole located at 1628, 1605, and 1585 of $\left(\mathrm{H}_{2} \mathrm{~L}-1\right)$ shift to lower frequencies that indicated their bonding to metal atom. The thioamide $v(\mathrm{C}=\mathrm{S})$ of $\mathrm{H}_{2} \mathrm{~L}-2$ located at $938 \mathrm{~cm}^{-1}$ shifted by $20-30 \mathrm{~cm}^{-1}$ in complexes of types $\left[\mathrm{M}\left(\mathrm{H}_{2} \mathrm{~L}-2\right) \mathrm{X}_{2}\right]$ indicating bonding of thione sulphur (Table 3). The $\nu(\mathrm{C}=\mathrm{S})$ shifts to $770-790 \mathrm{~cm}^{-1}$ in complexes 


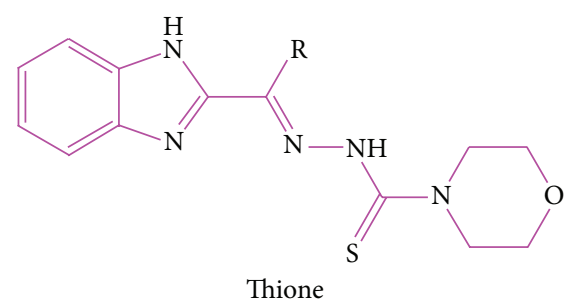

Figure 1: Figure of thione where $\mathrm{R}=\mathrm{CH}_{3}=\mathrm{H}_{2}$ bmctz and $\mathrm{R}=\mathrm{C}_{6} \mathrm{H}_{5}=\mathrm{H}_{2}$ bpmctz.

of types $\left[\mathrm{M}(\mathrm{HL}-2)_{2}\right]$ and $[\mathrm{M}(\mathrm{L}-2) \mathrm{B}]$ supporting bonding through deprotonated thiol sulphur of thioamide group [40, 41]. The I.R. spectral band of both $\mathrm{H}_{2} \mathrm{~L}-1$ and $\mathrm{H}_{2} \mathrm{~L}-2$ shows similar trend of bonding of donor atoms. Infar I.R. range, the I.R. spectra of complexes were recorded above $400 \mathrm{~cm}^{-1}$, therefore $v(\mathrm{M}-\mathrm{S})$ and $\nu(\mathrm{M}-\mathrm{N})$ stretches could not be located definitely. However, the new I.R. bands in complexes at 420$435 \mathrm{~cm}^{-1}$ are tentatively suggested to $v(\mathrm{M}-\mathrm{N})$ and $v(\mathrm{M}-\mathrm{S})$ vibrations (Table 3 ). In Finger print region $\mathrm{H}_{2} \mathrm{~L}-1, \mathrm{H}_{2} \mathrm{~L}-2$, and their complexes display a number of I.R. bands due to phenyl ring skeletal vibrations and $\delta\left(\mathrm{CH}_{2}\right), \delta\left(\mathrm{CH}_{3}\right), v(\mathrm{~N}-\mathrm{N})$, $\nu(\mathrm{C}-\mathrm{N})$, and $\nu(\mathrm{C}-\mathrm{C})$ vibrations as well as ring deformation vibrations [40, 41]. In general, almost all complexes display very strong band near $735-745 \mathrm{~cm}^{-1}$ attributed to phenyl ring out of plane bending of substituted phenyl ring. The Finger print region, I.R. stretches of $\mathrm{H}_{2} \mathrm{~L}-1$ are located at $1545,1515,1480,1441,1363,1271,1235,1155,1110,1004$, and $744 \mathrm{~cm}^{-1}$. These are retained in complexes with slight change in intensity and slight change in position [40-42]. The I.R. bands of $\mathrm{H}_{2} \mathrm{~L}-2$ were observed at $1540,1505,1482,1442,1336$, $1280,1240,1150,1105,1030,845$ and $742 \mathrm{~cm}^{-1}$. From the I.R. studies, it is concluded that the ligands are tridentate and dianionic in $[\mathrm{MLB}]$ type of complexes.

${ }^{1} \mathrm{H}$ NMR spectra of ligands $\mathrm{H}_{2} \mathrm{~L}-1$ and $\mathrm{H}_{2} \mathrm{~L}-2$ were recorded in DMSO at field strength $300 \mathrm{MHz}$. The ligand H2L-1 shows $\left(\mathrm{CH}_{2}\right)$ proton. Signals of morpholine ring as two triplets for $8 \mathrm{H}$ at $\delta=4.016,4.030,4.047$ and $4.154,4.170$, $4.184 \mathrm{ppm}$, the $\mathrm{CH}_{3}$, proton signals at $\delta=1.787 \mathrm{ppm}$ (Singlet), the phenyl ring signal at $\delta=6.843-6.914 \mathrm{ppm}$ as multiplet $(4 \mathrm{H})$. The benzimidazole ring $\mathrm{N}-\mathrm{H}$ and hydrazide part $\mathrm{NH}$ signals are located at $\delta=7.262$ and $\delta=8.6 \mathrm{ppm}$. The ligand $\mathrm{H}_{2} \mathrm{~L}-2$ displays $\left(\mathrm{CH}_{2}\right)$ proton signal of morpholine ring at $3.781-4.145 \mathrm{ppm}$ as two triplets $(8 \mathrm{H}, \mathrm{t})$ and phenyl ring $(\mathrm{C}-\mathrm{H})$ proton signal at $\delta=6.93-7.28$ as multiplet $(9 \mathrm{H})$. The benzimidazole ring $(\mathrm{NH})$ and hydrazide $(\mathrm{N}-\mathrm{H})$ proton signals were located at $\delta=7.415$ and $8.215 \mathrm{ppm}$. The mass spectrum of $\mathrm{H}_{2} \mathrm{~L}-$ 1 gave $M^{+} / z$ at 303(3\%), 288(3.8\%), 217(40\%), 117(100\%), and $86(30 \%)$. The calculated value of mass was 303 . The mass spectral results of $\mathrm{H}_{2} \mathrm{~L}-2$ indicated $\mathrm{M}^{+} / z$ values $365(5 \%)$, $288(3 \%), 279(8 \%), 86(30 \%)$, and $117(100 \%)$ as base peak. The mass calculated for $\mathrm{H}_{2} \mathrm{~L}-2$ was 365 . The ${ }^{1} \mathrm{HNMR}$ spectra of complexes could not be recorded due to their poor solubility (See Figure 3).

3.1. Antifungal Studies. The antifungal activity of ligands and some of their complexes have been evaluated by radial growth

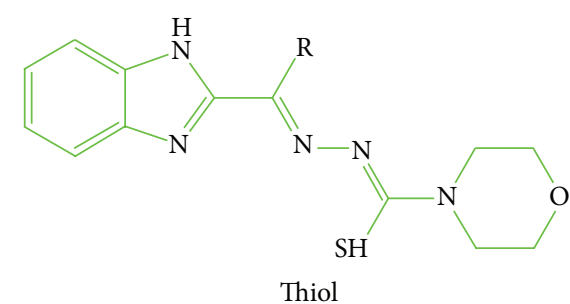

Figure 2: Figure of thiol where $\mathrm{R}=\mathrm{CH}_{3}=\mathrm{H}_{2}$ bmctz and $\mathrm{R}=\mathrm{C}_{6} \mathrm{H}_{5}=\mathrm{H}_{2}$ bpmctz.

method [43, 44]. Czapek agar medium was prepared by dissolving $20 \mathrm{~g}$ starch, $20 \mathrm{~g}$ agar agar and $20 \mathrm{~g}$ glucose in one litre distilled water. The resulting medium was added requisite amount of test compound to get 100 and $200 \mathrm{ppm}$ of solution. The resulting medium was then poured into Petri plates and the spores of fungi were placed on medium with the help of inoculum needle. The petri plates were wrapped in polythene bags containing two to three drops of ethanol and then placed in incubator at $30 \pm 0.5^{\circ} \mathrm{C}$. The linear growth of fungus was evaluated by measuring the fungal zone diameter after five days. The percentage inhibition was calculated from the relation $100 \times(C-T / C)$ where $C$ and $T$ are the diameter of the fungus colony and control test plate, respectively. The fungi used in present investigation for screening against the fungi, Aspergillus niger, Aspergillus flavus, F. oxysporum, Rhizoctonia bataticola, and R. phaseoli. The control solution was mycostatin. The result of activity is shown in Table 4. It has been found that the ligand containing phenyl substituent is more active than methyl substituent. The $\mathrm{Cu}$ (II) and $\mathrm{Zn}$ (II) complexes in general show larger activity than $\mathrm{Mn}$ (II) and $\mathrm{Fe}(\mathrm{II})$ complexes. The bromo complexes show larger activity than that of chloro or pydridine or $Y$-picoline adduct. The chemical Mancozeb fungicides were used as standard for this process. In general the antifungal activities observed for $\mathrm{Cu}$ (II) and $\mathrm{Zn}$ (II) halo complexes were much larger than other metal complexes and ligand. The antifungal activity observed for $R$. phaseoli is larger compared to other fungi, indicating selectivity of complexes for particular fungi. The enlarged activity of complexes may be attributed to increased delocalisation of $\pi$-electrons of ligand over the whole chelate ring which enhances the lipophilicity of the complexes. The increase lipophilicity enhances the penetration of complexes into fungi membrane and blocking the active binding sites in the microorganism. In addition the complexes disturb the respiration process of the fungi cell thus blocking the synthesis of proteins essential for fungi growth and restrict the further growth of the organism. The activity of complexes of phenyl substituted ligand is larger than methyl derivatives due to larger $\pi$-delocalised electron in phenyl substituted ligand than that of methyl derivatives and free ligand as well.

In addition to above facts, the chelate formation reduces the polarity of metal ions, increases the lipophilic character of the chelate, and increases the interaction between metal ion and fungi cell membrane. Consequently it blocks the cell growth of microorganism. According to overtone's concept the lipophilicity of complexes is major factor which favours 


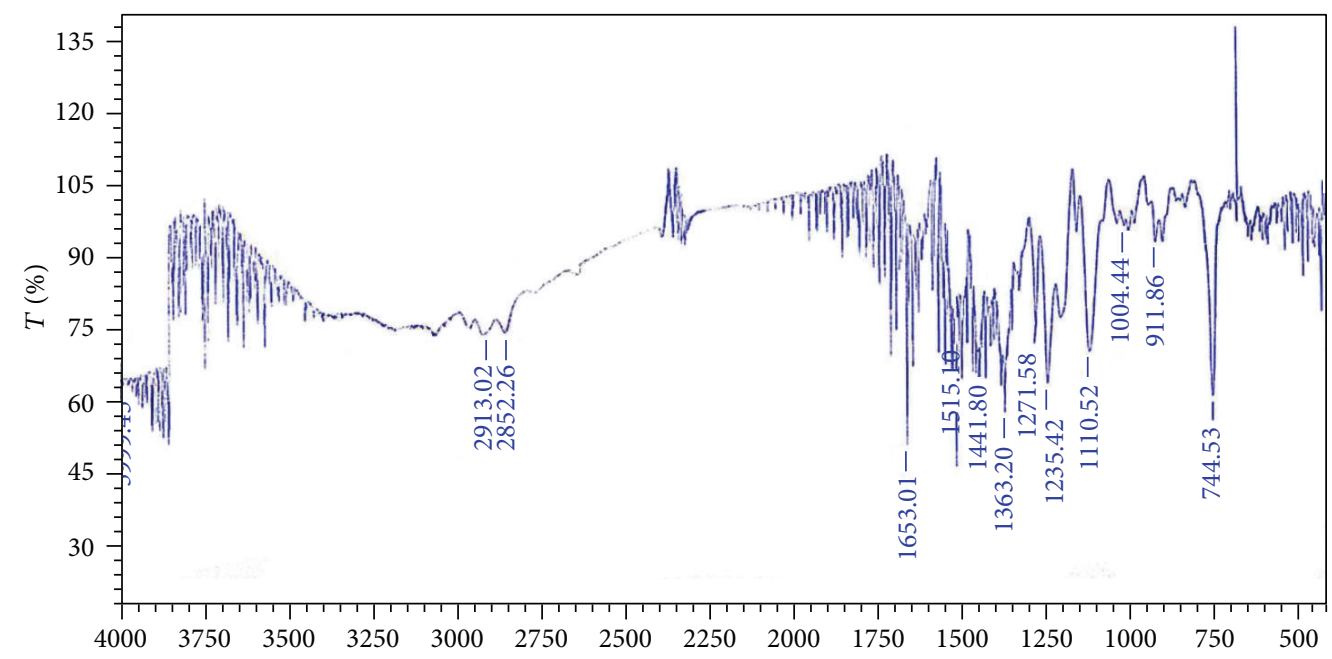

FIGURE 3: Graph of I.R. spectra of morpholine-N-carbothiohydrazide of 2-acetylbenzimidazole $\left(\mathrm{H}_{2}\right.$ bmctz).

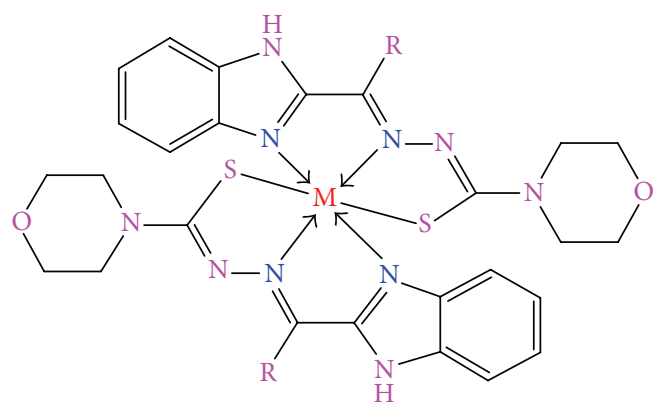

Figure 4: Proposed structure of $\left[\mathrm{M}(\mathrm{HL})_{2}\right]$ where $\mathrm{M}=\left(\mathrm{Co}^{\mathrm{II}}, \mathrm{Ni}^{\mathrm{II}}\right.$ $\mathrm{HL}=\mathrm{Hbmctz}$ or $\mathrm{HL}=$ Hbpmctz.<smiles>[X][M]C1c2ccccc2NC1C([R])=NNC(=S)N1CCOCC1</smiles>

Figure 5: Proposed structure of $\left[\mathrm{M}\left(\mathrm{H}_{2} \mathrm{~L}\right) \mathrm{X}_{2}\right]$ type of complexes where $\mathrm{M}=\mathrm{Zn}^{\mathrm{II}}, \mathrm{Cu}^{\mathrm{II}}, \mathrm{Ni}^{\mathrm{II}}, \mathrm{Cd}^{\mathrm{II}}$, and $\mathrm{X}=\mathrm{Cl}^{-}$or $\mathrm{Br}^{-}$.

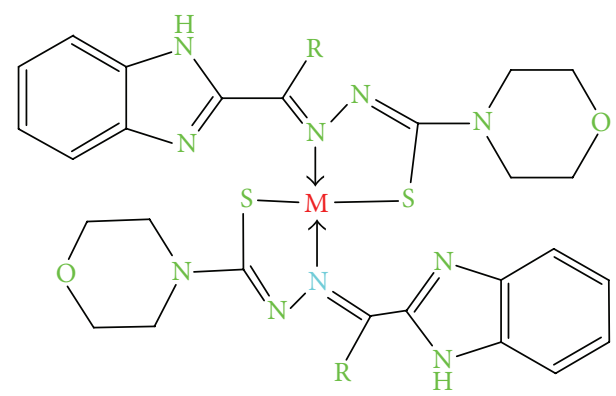

FIGURE 6: Tetrahedral structure for $\mathrm{Zn}^{\mathrm{II}}$ or $\mathrm{Cd}^{\mathrm{II}}$, square planar for $\mathrm{Ni}^{\mathrm{II}}$ or $\mathrm{Pd}^{\mathrm{II}}$.

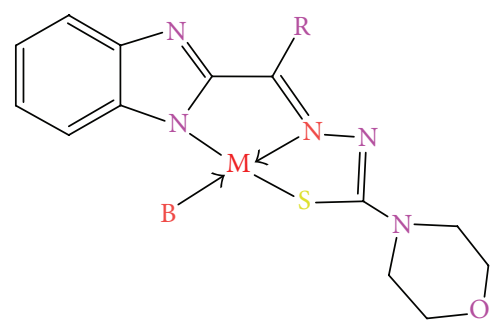

FIgURE 7: Structure of [MLB] where $\mathrm{M}=\mathrm{Ni}^{\mathrm{II}}, \mathrm{Cu}^{\mathrm{II}}$ are planar but tetrahedral for $\mathrm{Zn}^{\mathrm{II}}, \mathrm{Cd}^{\mathrm{II}} \mathrm{H}_{2} \mathrm{~L}=\mathrm{H}_{2}$ bmctz or $\mathrm{H}_{2}$ bpmctz, $\mathrm{B}=\mathrm{Py}, \mathrm{H}_{2} \mathrm{O}$ and $Y$-Pic.

antimicrobial activity $[45,46]$. The hydrogen bond formation between fungi and complex molecule through azomethene $(\mathrm{C}=\mathrm{N})$ and heteroatoms $\mathrm{S}$ and $\mathrm{O}$ and halogens of complexes interacts with cell constituents resulting in interference with the normal cell process. These factors are tentatively suggested for enhanced antimicrobial activities of metal chelates complexes of $\mathrm{N}$ and $\mathrm{S}$ donor.

From the results of I.R. and physical data, the probable structures of complexes are shown in Figures 4, 5, 6, and 7.

\section{Conclusion}

The physicochemical data suggested octahedral structure for $\left[\mathrm{M}(\mathrm{HL})_{2}\right]$ type of complexes of $\mathrm{Ni}(\mathrm{II}), \mathrm{Co}(\mathrm{II})$, and $\mathrm{Cu}(\mathrm{II})$ while four coordinated planar to Pd(II) complexes. The monoligated $\mathrm{Ni}(\mathrm{II})$ and $\mathrm{Pd}(\mathrm{II})$ complexes [M(L-1)/(1-2)B] show characteristics of planar geometry. The complexes of $\left[\mathrm{M}\left(\mathrm{H}_{2} \mathrm{~L}\right) \mathrm{Cl}_{2}\right],\left(\mathrm{M}=\mathrm{Co}^{\mathrm{II}}, \mathrm{Ni}^{\mathrm{II}}, \mathrm{Cu}^{\mathrm{II}}, \mathrm{Zn}^{\mathrm{II}}\right.$ or $\left.\mathrm{Cd}^{\mathrm{II}}\right)$ have been suggested to possess trigonal bipyramidal structures. The ligand $\mathrm{H}_{2} \mathrm{~L}-1$ and $\mathrm{H}_{2} \mathrm{~L}-2$ coordinate as neutral, monoanionics or dianionic tridentate $(\mathrm{N}, \mathrm{N}, \mathrm{S})$ donor molecules. The biological activity of phenyl substituted ligand and its complexes are larger than those of methyl substituted products probably due 
to excessive delocalized $\pi$-electron system present in phenyl ring.

\section{Conflict of Interests}

The authors declare that there is no conflict of interests regarding the publication of this paper.

\section{Acknowledgments}

Thanks are due to authority of IIT Patna for recording IR, U$\mathrm{V}$, and mass spectra, BIT Mesra Ranchi for C, H, or N analysis, and CDRI Lucknow for ${ }^{1} \mathrm{H}$ NMR spectra, and the Head of the Chemistry Department, Patna for magnetic susceptibility measurements. The authority of NIT Patna is thankful for providing necessary laboratory facilities. We thankfully acknowledge the help of Biotechnology Department, Science College Patna, for screening antifungal activity.

\section{References}

[1] S. O. Podunavac-Kuzmanovic, D. M. Cvetkovic, and L. S. Vojinovic, "Synthesis, physico-chemical characterization and biological activity of 2-aminobenzimidazole complexes with different metal ions," APTEFF, vol. 35, pp. 239-246, 2004.

[2] S. P. Ghosh and L. K. Mishra, "Complex compounds of iron(II, III) with 2-(2' -pyridyl)benzimidazol," Inorganica Chimica Acta, vol. 7, pp. 545-548, 1973.

[3] S. P. Ghosh, P. Bhattacharjee, L. Dubey, and L. K. Mishra, "Complexes of some platinum metals with imidazole and benzimidazole," Journal of the Indian Chemical Society, vol. 52, pp. 230-235, 1974.

[4] S. K. Gupta and L. K. Mishra, "Oxo-vanadium(IV) complexes with 2-(o-hydroxyphenyl)benzimidazole and related ligands," Journal of Inorganic and Nuclear Chemistry, vol. 41, no. 6, pp. 890-891, 1979.

[5] K. K. R. Anil and L. K. Mishra, "Complexes of Rh(III) and Pd(II) with $\mathrm{N}$-vinylimidazole," Journal of the Indian Chemical Society, vol. 64, pp. 503-505, 1987.

[6] M. Bala, K. Ahmad, S. Sharma, and L. K. Mishra, "Preparation, characterisation and anti-fungal activity of $1 \mathrm{E}-1-(1 \mathrm{H}-$ benzimidazol-2-yl)-N-hydroxy ethanimine and 1E-1-(1Hbenzimidazol-2-yl)-N-hydroxy-1-phenyl methanimine withCo(II), $\mathrm{Ni}(\mathrm{II}), \mathrm{Cu}(\mathrm{II}), \mathrm{Zn}(\mathrm{II})$ and $\mathrm{Cd}(\mathrm{II}), "$ IOSR Journal of Applied Chemistry, vol. 3, no. 2, pp. 21-29, 2012.

[7] M. Bala and L. K. Mishra, "Synthesis, characterisation and anti amoebic activity of 1E-1-(1H-benzimidazole-2-yl)-N-hydroxy ethanimine and 1E-1-(1H-benzimidazole-2-yl)-N-hydroxy-1phenyl methanimine with oxovanadium (IV), Mn(II), Fe(II)," International Journal of Emerging Technologies in Computationaland Applied Sciences, vol. 4, no. 4, pp. 347-353, 2013.

[8] A. K. Tripathi and P. Mathur, "A binuclear iron(III) complex of $\mathrm{N}, \mathrm{N}, \mathrm{N}^{\prime}, \mathrm{N}^{\prime}$-tetrakis[(2-benzimidazolyl)]-1,2-ethanediamine," Journal of the Indian Chemical Society, vol. 70, p. 991, 1993.

[9] S. Das, S. Malik, and B. Jain, "Synthesis and spectroscopic characterization of metal complexes of rabeprazole drug," Journal of Indian Council of Chemist, vol. 27, no. 2, pp. 177-179, 2010.
[10] M. R. Maurya, S. Sikarwar, T. Joseph, P. Manikandan, and S. B. Halligudi, "Synthesis, characterization and catalytic potentials of polymer anchored copper(II), oxovanadium(IV) and dioxomolybdenum(VI) complexes of $2-(\alpha-$ hydroxymethyl)benzimidazole," Reactive and Functional Polymers, vol. 63, no. 1, pp. 71-83, 2005.

[11] B. E. Evans, K. E. Rittle, M. G. Bock et al., "Methods for drug discovery: development of potent, selective, orally effective cholecystokinin antagonists," Journal of Medicinal Chemistry, vol. 31, no. 12, pp. 2235-2246, 1988.

[12] A. A. Spasov, I. N. Yozhitsa, L. I. Bugaeva, and V. A. Anisimova, "Benzimidazole derivatives: spectrum of pharmacological activity and toxicological properties (a review)," Pharmaceutical Chemistry Journal, vol. 33, no. 5, pp. 232-243, 1999.

[13] H. A. Barker, R. D. Smyth, H. Weissbach, J. I. Toohey, J. N. Ladd, and B. E. Volcani, "Isolation and properties of crystalline cobamide coenzymes containing benzimidazole or 5, 6dimethylbenzimidazole," The Journal of Biological Chemistry, vol. 235, pp. 480-488, 1960.

[14] H. Nakano, T. Inoue, N. Kawaohaki et al., "Synthesis and biological activities of novel antiallergic agents with 5-lipoxygenase inhibiting action," Bioorganic \& Medicinal Chemistry, vol. 8, no. 2, pp. 373-380, 2000.

[15] K. Starčević, M. Kralj, K. Ester et al., "Synthesis, antiviral and antitumor activity of 2-substituted-5-amidinobenzimidazoles," Bioorganic \& Medicinal Chemistry, vol. 15, no. 13, pp. 4419-4426, 2007.

[16] G.-F. Wang, L.-P. Shia, Y.-D. Ren et al., "Anti-hepatitis B virus activity of chlorogenic acid, quinic acid and caffeic acid in vivo and in vitro," Antiviral Research, vol. 83, no. 2, pp. 186-190, 2009.

[17] Y.-F. Li, G.-F. Wang, P.-L. He et al., "Synthesis and anti-hepatitis B virus activity of novel benzimidazole derivatives," Journal of Medicinal Chemistry, vol. 49, no. 15, pp. 4790-4794, 2006.

[18] K. Ishihara, T. Ichikawa, Y. Komuro, S. Ohara, and K. Hotta, "Effect on gastric mucus of the proton pump inhibitor leminoprazole and its cytoprotective action against ethanol-induced gastric injury in rats," Drug Research, vol. 44, no. 7, pp. 827-830, 1994.

[19] W. A. Maxwell and G. Brody, "Antifungal activity of selected benzimidazole compounds," Applied Microbiology, vol. 21, no. 5, pp. 944-945, 1971.

[20] P. L. Beaulieu, M. Bös, Y. Bousquet et al., "Non-nucleoside inhibitors of the hepatitis C virus NS5B polymerase: discovery and preliminary SAR of benzimidazole derivatives," Bioorganic \& Medicinal Chemistry Letters, vol. 14, no. 1, pp. 119-124, 2004.

[21] P. T. M. Nguyen, J. D. Baldeck, J. Olsson, and R. E. Marquis, "Antimicrobial actions of benzimidazoles against oral streptococci," Oral Microbiology and Immunology, vol. 20, no. 2, pp. 93-100, 2005.

[22] M. Boiani and M. Gonzalez, "Imidazole and benzimidazole derivatives as chemotherapeutic agents," Mini-Reviews in Medicinal Chemistry, vol. 5, no. 4, pp. 409-424, 2005.

[23] H. Küçükbay, R. Durmaz, E. Orhan, and S. Günal, "Synthesis, antibacterial and antifungal activities of electron-rich olefins derived benzimidazole compounds," Il Farmaco, vol. 58, no. 6, pp. 431-437, 2003.

[24] V. K. Pandey, M. Upadhyay, M. Upadhyay, V. D. Gupta, and M. Tandon, "Benzimidazolyl quinolinyl mercaptotriazoles as potential antimicrobial and antiviral agents," Acta Pharmaceutica, vol. 55, no. 1, pp. 47-56, 2005.

[25] D. Y. Graham, A. McCullough, M. Sklar et al., "Omeprazole versus placebo in duodenal ulcer healing. The United States 
experience," Digestive Diseases and Sciences, vol. 35, no. 1, pp. 66-72, 1990.

[26] P. A. Janssen and F. T. Allewijn, "Pimozide, a chemically novel, highly potent and orally long-acting neuroleptic drug. II. Kinetic study of the distribution of pimozide and metabolites in brain, liver, and blood of the Wistar rat," Drug Research, vol. 18, no. 3, pp. 279-282, 1968.

[27] B. Halliwell and J. M. C. Gutteridge, "Role of free radicals and catalytic metal ions in human disease: an overview," Methods in Enzymology, vol. 186, pp. 1-85, 1990.

[28] W. N. Lipscomb and N. Sträter, "Recent advances in zinc enzymology," Chemical Reviews, vol. 96, no. 7, pp. 2375-2434, 1996.

[29] D. R. Richardson, "Iron chelators as therapeutic agents for the treatment of cancer," Critical Reviews in Oncology/Hematology, vol. 42, no. 3, pp. 267-281, 2002.

[30] M. E. Maguire and J. A. Cowan, "Magnesium chemistry and biochemistry," BioMetals, vol. 15, no. 3, pp. 203-210, 2002.

[31] R. R. Crichton, Biological Inorganic Chemistry: An Introduction, Elsevier, Amsterdam, The Netherlands, 2008.

[32] M. Sugiyama, "Role of cellular antioxidants in metal-induced damage," Cell Biology and Toxicology, vol. 10, no. 1, pp. 1-22, 1994.

[33] K. Ramaiah, J. S. Grossert, D. L. Hooper, P. K. Dubey, and J. Ramanatham, "Studies on synthesis of 2-acetylbenzimidazole and related benzimidazole derivatives," Journal of the Indian Chemical Society, vol. 76, no. 3, pp. 140-144, 1999.

[34] L. F. Audrieth, E. S. Scott, and P. S. Kippur, "Hydrazine derivatives of the carbonic and thiocarbonic acids. I. The preparation and properties of thiocarbohydrazide," Journal of Organic Chemistry, vol. 19, no. 5, pp. 733-741, 1954.

[35] M. A. Phillips, "CCCXVII.-the formation of 2-substituted benziminazoles," Journal of the Chemical Society, pp. 2393-2399, 1928.

[36] W. J. Geary, "The use of conductivity measurements in organic solvents for the characterisation of coordination compounds," Coordination Chemistry Reviews, vol. 7, no. 1, pp. 81-122, 1970.

[37] B. N. Figgis and J. Lewis, "The magnetic properties of transition metal complexes," in Progress in Inorganic Chemistry, vol. 6, pp. 37-239, John Wiley \& Sons, New York, NY, USA, 1964.

[38] R. C. Stoufer, D. W. Smith, E. A. Clevenger, and T. E. Norris, "Complexes of cobalt(II). I. On the anomalous magnetic behavior of some six-coordinate cobalt(II) complexes," Inorganic Chemistry, vol. 5, no. 7, pp. 1167-1171, 1966.

[39] A. B. P. Lever, Inorganic Electronic Spectroscopy, Elsevier, New York, NY, USA, 1968.

[40] M. K. Das, M. Nath, and J. J. Zuckerman, "Di- and triorganotin(IV) derivatives of N,N-substituted hydroxylamines," Inorganica Chimica Acta, vol. 71, pp. 49-59, 1983.

[41] M. R. Gajendragad and U. C. Agrawala, "Complexing behaviour of 5-amino-1,3,4-thiazole-2-thiol. II. Complexes of Ni(II), $\mathrm{Rh}(\mathrm{I}), \mathrm{Pd}(\mathrm{II}), \mathrm{Pt}(\mathrm{II}), \mathrm{Au}(\mathrm{III})$ and $\mathrm{Cu}(\mathrm{II})$, Bulletin of the Chemical Society of Japan, vol. 48, no. 3, pp. 1024-1029, 1975.

[42] K. Nakamoto, Infrared and Raman Spectra of Inorganic and Complex Compounds, John Wiley \& Sons, New York, NY, USA, 1978.

[43] A. W. Bauer, W. M. Kirby, J. C. Sherris, and M. Turck, "Antibiotic susceptibility testing by a standardized single disk method," American Journal of Clinical Pathology, vol. 45, no. 4, pp. 493496, 1966.
[44] F. Cavanagh, Analytical Microbiology, Academic Press, New York, NY, USA, 1963.

[45] Y. Anjaneyulu, L. N. Murthy, and R. P. Rao, "Preparation, characterizationand antimicrobial activity studies on some ternary complexes of $\mathrm{Cu}$ (II) with acetylacetone and various salicylic acids," Synthesis and Reactivity in Inorganic and MetalOrganic Chemistry, vol. 16, no. 2, pp. 257-272, 1986.

[46] T. J. Franklin and G. A. Snow, "Penetrating the defences," in Biochemistry of Antimicrobial Action, Chapman and Hall, London, UK, 1971. 

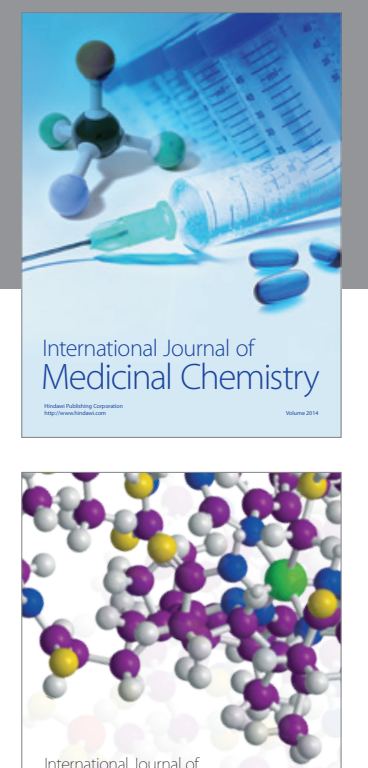

\section{Carbohydrate} Chemistry

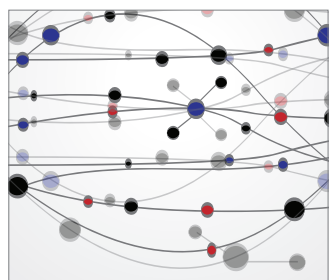

The Scientific World Journal
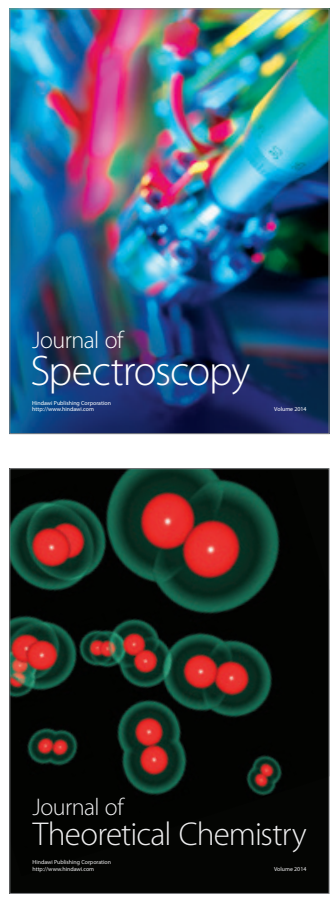
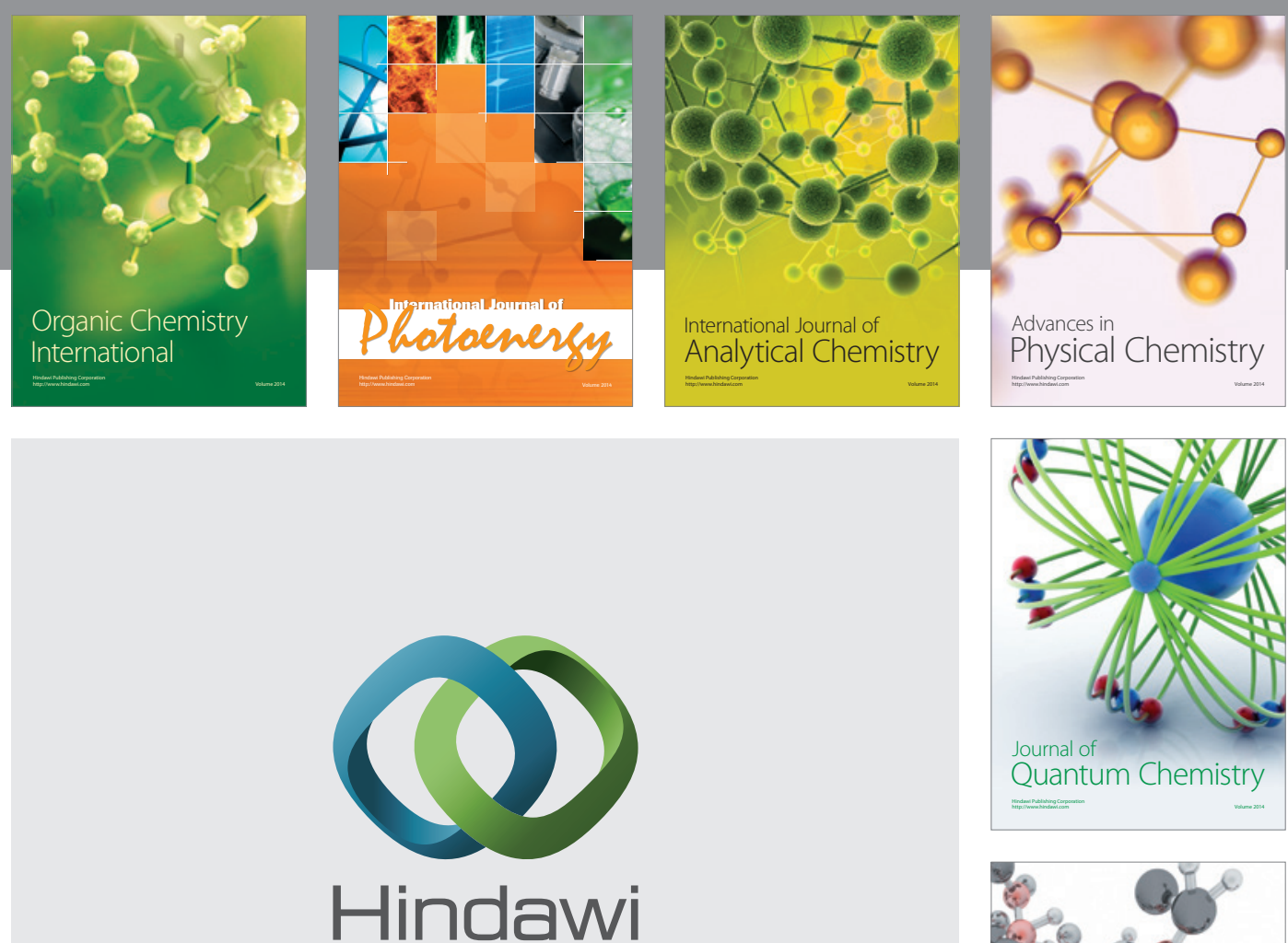

Submit your manuscripts at

http://www.hindawi.com

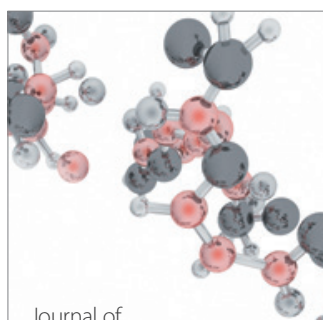

Analytical Methods

in Chemistry

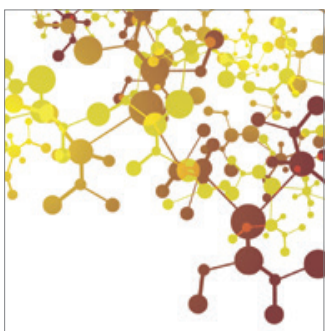

Journal of

Applied Chemistry

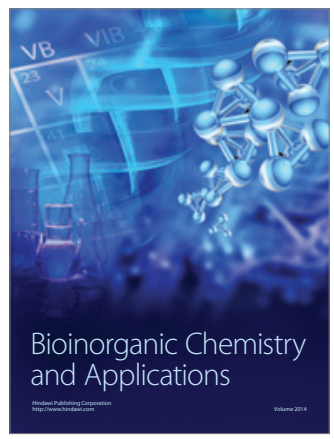

Inorganic Chemistry
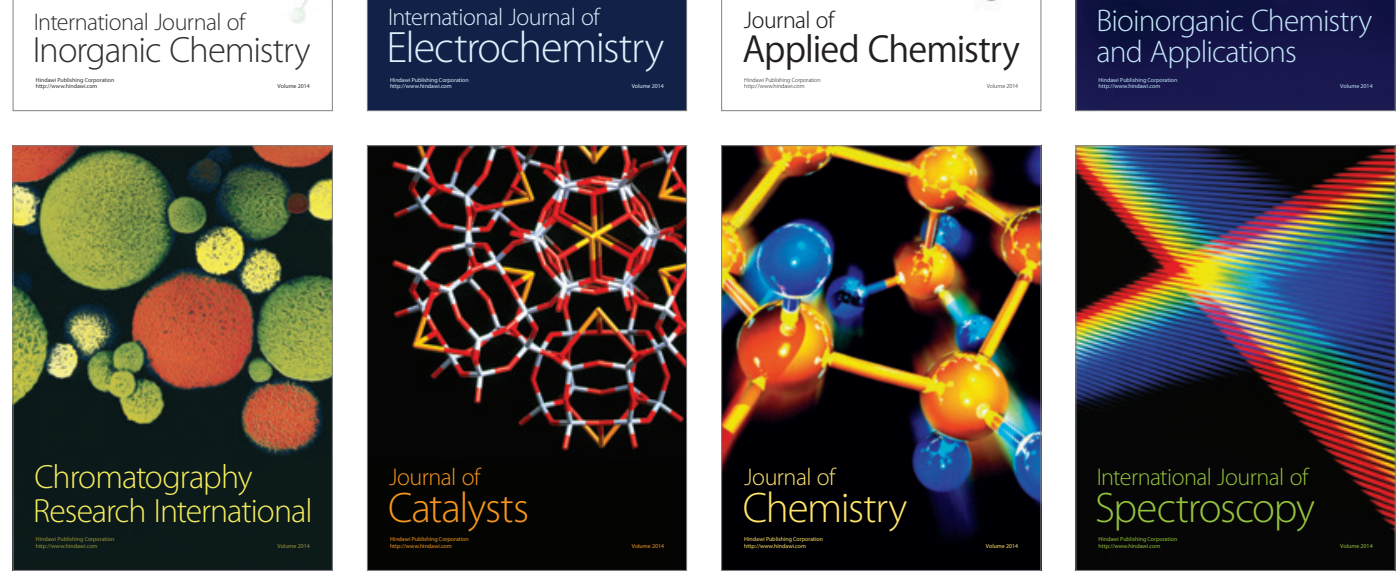